$$
\text { DOE/PC/79903- TII }
$$

\title{
OPTICAL PROPERTIES OF FLYASH
}

\author{
Contract No. DE-AC22-87PC 79903
}

Quarterly Report for Period 1 October - 31 December 1991

Prepared for Pittsburgh Energy Technology Center

Principal Investigator Professor S. A. Self

A 11809982

January 1992

\section{HIGH TEMPERATURE GASDYNAMICS LABORATORY Mechanical Engineering Department Stanford University}




\section{OPTICAL PROPERTIES OF FLYASH}

Contract No. DE-AC22-87PC 79903

Quarterly Report for Period 1 October - 31 December 1991

Prepared for Pittsburgh Energy Technology Center

Principal Investigator Professor S. A. Self

January 1992

\section{DISCLAIMER}

This report was prepared as an account of work sponsored by an agency of the United States This report was propared as an account of work sponsor any agency thereof, nor any of their Government. Neither the United States Government nor any agency the liability or responsiemployees, makes any warranty, express or implied, or assuinformation, apparatus, product, or bility for the accuracy, completeness, or usefulness of any information, apparatus, product, process disclosed, or represents that its use would not infringe privately owned righe, trademark, ence herein to any specific commercial product, process, or service by trade name, tradent, recommanufacturer, or otherwise does not necessarily constitute or imply its endorsement, The views mendation, or favoring by the United States Government or any agency thereof. The views and opinions of authors expressed herein do not

United States Government or any agency thereof. 


\section{OPTICAL PROPERTIES OF FLYASH \\ Contract No. DE-AC22-87PC 79903 \\ Quarterly Report for Period 1 October - 31 December 1991 \\ Prepared for Pittsburgh Energy Technology Center \\ Principal Investigator Professor S. A. Self}

\section{EXECUTIVE SUMMARY}

The general aims of this research are to provide a fundamental scientific basis for the physical understanding and reliable calculation of radiative heat transfer in coal combustion systems, particularly as it is influenced by the presence of inorganic constituents deriving from the mineral matter in coal.

The work is organized under four tasks. Tasks I and II were initiated in October 1987; Tasks III and IV were funded from October 1988.

Task 1. Characterization of Flyash: Under this heading the chemical composition and size distribution of representative flyashes are being measured by appropriate microanalytical techniques to provide information required in Task 2.

Task 2. Measurements of the Optical Constants of Slags: Under this heading measurements of the infrared optical constants (i.e., the complex refractive index $m=n-i k$ ) of synthetic slags are being made as a function of wavelength and temperature for controlled compositions. Particular attention will be given to the contribution of the $\mathrm{Fe}_{2} \mathrm{O}_{3}$ content and its valence state. The data is being reduced to yield formulae giving the complex refractive index over relevant ranges of wavelength and temperature, as a function of the relevant metal oxide constituents.

Task 3. Sample Calculations of the Radiant Properties of Flyash Dispersions: This component comprises various calculations to guide and evaluate the experimental work under the other three tasks.

Task 4. Measurement of the Radiant Properties of Flyash Dispersions: This benchscale experiment is planned to compare the measured radiant properties of a dispersion of well-characterized ash with computations based on data developed under the first two tasks.

In this seventeenth quarter good progress has been made under Tasks 2 and 4, as reported in the Quarterly Report, and summarized below. Tasks 1 and 2 are now essentially complete apart from writing of a comprehensive report. Some further computations are planned under Task 3 while a series of measurements remain to be made under Task 4 . 


\section{Task 1}

The ashes being characterized are samples from power plants or pilot-scale combustors derived from combustion of six of the coals selected for study under the parallel PETC program on "Transformation of Inorganic Coal Constituents in Combustion Systems."

The principal features requiring characterization are particle size and composition distributions, including correlations between size and composition. Size distributions are being measured in house by Coulter counter. Size and composition distributions are being determined by automated SEM/microprobe analysis at UNDERC. Other in-house characterization work under way includes size classification by wet sieving combined with classification by density using flotation/sedimentation techniques, low temperature ashing for the char content and magnetic separation for the magnetite content resulting from combustion of pyrite.

Size distributions for all six ashes over the range $0.5-60 \mu \mathrm{m}$ have been completed using the Coulter multisizer with a technique employing two orifices to cover the whole range. A suitable technique for matching distributions using two orifice sizes was devised. The measured distribution was found to be very well represented by truncated log-normal distributions. Comparison with the size distribution from automated SEM shows some discrepancy which is still being investigated.

Micropraphs of the ash samples prepared by UNDERC for automated SEM/EDX sizecomposition analysis revealed many agglomerates, casting some doubt on the validity of the data. A freeze-drying technique for sample preparation has been developed at Stanford which gives very well dispersed samples. The automated SEM/EDX analysis has been repeated using such samples and the results have been analyzed using statistical computer codes developed at Stanford. Ternary diagrams of the composition distributions (in color) have been prepared using software developed at Stanford. In the past quarter, automated SEM/EDX analyses have been completed and analysis of the data made for all six ashes.

In addition, density classification results have been obtained for all six ashes and compared with results from SEM analysis for composition, which, of course, determines the density. Currently, size distributions are being determined for the density-separated fractions to obtain composition/size correlations for comparison with the SEM/EDX data. This task is now essentially completed. 


\section{Task 2}

Methods for determining the infrared optical properties of solid synthetic and natural slags at low temperatures have been established in prior work at Stanford. In the present work the main effort has been devoted to the development of suitable apparatus and techniques for performing similar measurements on slags at temperatures to $2000 \mathrm{~K}$, when the slag is liquid. Basic experimental strategies have been decided and apparatus has been designed to accomplish this task. Tests at high temperatures during past quarters have resulted in the first reliable measurements of the infrared absorption of liquid slag.

Two complementary techniques involving infrared optical measurements on liquid samples of synthetic slag maintained in an electric furnace have been developed. The first, for the wavelength range $1-5 \mu \mathrm{m}$ where the absorption index is low $\left(\mathrm{k} \leq 10^{-2}\right)$ employs a submerged platinum mirror to measure the absorption of thin films of slag by a double-pass technique. The second, applicable over the whole wavelength range $(1-12 \mu \mathrm{m})$, measures the surface reflectance of the liquid slag relative to that of a cold gold mirror in an external reference path.

The first technique has been successfully used to obtain the first reliable measurements of the infrared absorption of liquid slag (at $2000 \mathrm{~K}$ ).

Efforts have since been concentrated on developing and testing the second technique (for surface reflectivity measuremenis). It was determined that the quality of the data obtained is limited by the differing absorptions due to $\mathrm{CO}_{2}$ and $\mathrm{H}_{2} \mathrm{O}$ in the hot measurement path and the cold reference path. To eliminate this problem, the whole apparatus has been enclosed in a chamber, purged with dry nitrogen.

With this modification, good measurements of the reflectivity of synthetic slag containing $5 \% \mathrm{Fe}$ at $1600^{\circ} \mathrm{C}$ were made over the whole wavelength range $1-12 \mu \mathrm{m}$. The data in the range $8-12 \mu \mathrm{m}$ were reduced, using the Kramers-Kronig technique to give both the real and imaginary parts of the complex refractive index.

Possible sources of uncertainty in these measurements have been critically investigated and resolved. In particular, a problem due to contamination of a mirror by furnace gases has been identified and steps taken to eliminate it by redesign of the optical system. Reflectance measurements on liquid slags containing zero, 1\%, 5\%, 10\% and $20 \%$ iron (as $\mathrm{Fe}_{2} \mathrm{O}_{3}$ ) have been completed. The results have been reduced, using Kramers-Kronig analysis to yield the real $(n)$ and imaginary $(k)$ parts of the complex refractive index.

Measurements are currently underway on liquid slags of varying $\mathrm{SiO}_{2}$ composition. When completed Task 2 will be essentially completed. 


\section{Task 3}

Programs have been written for Mie scattering calculations which are then convolved with input on the size and optical constants distributions for a particulate dispersion to yield the spectral scattering and absorption coefficients of the aerosol. Additionally, a program has been written to solve the radiation transfer problem for a homogeneous slab, utilizing the exact solution method of Case's normal modes. Input for the spectral scattering and absorption coefficients from the first program allows the spectral scattering, absorption and emission properties of the slab to be computed. These can then be integrated over wavelength to yield the total radiative heat transfer characteristics of the slab.

These programs have been used to determine the importance of certain features of typical ashes for radiation transfer. These include the sensitivity of the optical/radiative properties of a flyash dispersion to (i) composition-size correlation, especially with regard to the distribution of iron oxides with particle size, and (ii) the presence of bubbles in the glassy ash particles.

This computational capability is also being used to evaluate the experimental conditions in the design of the apparatus for Task 4.

\section{Task 4}

Careful consideration has been given to the feasibility of various basic approaches for implementing the goals of this task. After evaluating various experimental techniques, a basic approach has been identified, which involves extinction measurements on flyash dispersed in suitable organic liquids. Measurements of the infrared transmission of three selected liquids have been made which confirm their suitability for this purpose. $\mathrm{CaF} 2$ windows for an absorption cell have been acquired and preliminary tests of a suitable cell design have been made.

During the past quarter, careful consideration has been given to the detailed optical design of a transmissometer for measurements on flyash dispersions in infra-red transmitting liquids.

Now that Task 2 is completed, the infra-red optical system is being re-configured for the experimental phase of Task 4 . 


\subsection{INTRODUCTION}

This is the seventeenth quarterly report under DOE contract No. DE-AC22-87PC 79903 entitled "Optical Properties of Flyash." Tasks 1 and 2 of this program were funded from 15 September 1987. Tasks 3 and 4 were funded from 15 September 1988.

The general aims of this research are to provide a fundamental scientific basis for the physical understanding and reliable calculation of radiative heat transfer in coal combustion systems, particularly as it is influenced by the presence of inorganic constituents deriving from the mineral matter in coal. Some preliminary work in this area has been carried out at Stanford in the past several years with NSF support. The present program will greatly enlarge the scope of this work.

The complete, integrated program of theoretical and experimental work comprises four separate tasks.

Task 1. Characterization of Flyash

Task 2. Measurements of the Optical Constants of Slags

Task 3. Sample Calculations of the Radiant Properties of Flyash Dispersions.

Task 4. Measurements of the Radiative Properties of Flyash Dispersions.

In Task 1, the chemical composition and size distribution of representative flyashes are being measured by appropriate microanalytical techniques to provide information required in Tasks 2 and 3.

In Task 2, measurements of the infrared optical constants (i.e., the complex refractive index $m=n-i k$ ) of synthetic slags are being made as a function of wavelength and temperature for controlled compositions. Particular attention is being given to the contribution of $\mathrm{Fe}_{2} \mathrm{O}_{3}$ content and its valence state. The data will be reduced to yield formulae giving the complex refractive index over relevant ranges of wavelength and temperature, as a function of the relevant metal oxide constituents.

In Task 3, sample calculations are being made for typical ash loadings, size distributions and compositions for simple geometries, with two main purposes: first, to provide insight and physical understanding of the role of flyash in radiative heat transfer in combustion systems; second, to indicate the sensitivity of the results to the characteristics of the input data. Such calculations will also be used to determine appropriate conditions and to predict the expe ted measured radiative properties for the experiment of Task 4. 
The experiment of Task 4 is designed to critically test our ability to predict the measured spectral emittance and scattering coefficient of flyash dispersions under wellcontrolled laboratory conditions utilizing the optical property data developed in Task 2. Particular attention will be paid to assessing the contribution of the char component in typical ashes. Any discrepancies between calculated and measured quantities revealed by these tests will be resolved by appropriate further studies.

A more detailed description of the scope of these tasks is given below. First, however, an outline is given of the rationale for the overall approach adopted in this program.

\subsection{Rationale of Overall Approach}

To account for the effects of flyash in radiative heat transfer calculations requires a knowledge of the contributions of the ash to the spectral absorption $\left(a_{\lambda}\right)$ and scattering $\left(\sigma_{\lambda}\right)$ coefficients of the particulate dispersion, together with the phase function $\Phi_{\lambda}$ describing the anisotropy of the scattering. These quantities depend on the particulate loading as well as the distributions of the size and optical properties of the particles.

For a spherical particle of homogeneous, optically isotropic material, characterized by a complex refractive index $m \equiv(n-i k)$ Mie theory allows one to cornpute the spectral absorption $\left(Q_{\lambda, \mathrm{a}}\right)$ and scattering $\left(Q_{\lambda, \mathrm{s}}\right)$ efficiencies of the particle, as well as the phase function $\phi_{\lambda}$. For randomly polarized radiation, these quantities are a function of the particle size parameter $x \equiv(\pi \mathrm{d} / \lambda)$, and the complex refractive index $m(C, \lambda, T)$, a function of composition, wavelength and temperature.

For a monodispersion of identical spherical particles, of specified loading (i.e. number density), the particulate's contribution to the optical properties $\left(a_{\lambda}, \sigma_{\lambda}\right.$ and $\left.\Phi_{\lambda}\right)$ of the medium are simply related to the spectral properties $\left(Q_{\lambda, a}, Q_{\lambda, s}, \phi_{\lambda}\right)$ of a single particle. It is also straightforward to compute the spectral optical properties of the medium for a polydispersion of spheres of identical composition, by convolving the results of Mie calculations for spheres of varying diameter (i.e. $x$ ) for fixed wavelength (and hence fixed $\mathrm{m}$ ), with the particle size distribution (assumed given). In the case of a particulate material, like flyash, for which it is reasonable to assume that individual particles are of homogeneous composition but the composition varies from particle to particle, it is still possible to compute the spectral characteristics of the particulate dispersion by dividing the particles into an appropriate number of classes of varying composition (and hence $\mathrm{m}$ ), each having a specified size distribution, and summing over particle classes. 
In radiative heat transfer calculations, the contribution of the gas to the spectral absorption coefficient is added to that of the particles to obtain the combined optical properties of the medium on a spectral basis. These optical properties are then used as inputfor a radiation transfer code to calculate radiative fluxes, on a spectral basis, for a particular combustor geometry and boundary conditions. Finally, to obtain total heat transfer quantities such as the overall radiant heat flux, integrations over wavelength must be made.

The procedure, outlined above, represents the only logical approach to the computation of radiative heat transfer in flyash laden combustion gases. To implement this procedure requires, as input, a detailed characterization of the ash with respect to its size and (complex) refractive index distributions on a spectral basis.

Now, while techniques are available for determining the size distribution of powder samples, such as flyash, there are no practical means available for reliably determining the complex refractive index distribution of a complex material such as flyash either on a single particle basis, as a powder or as a dispersed aerosol. However, it is possible, using modern microanalytical techniques, specifically computer-automated SEM/EDX analysis, to determine the size and chemical composition of a heterogeneous powder on a particle by particle basis for a statistically large number of particles.

If the compositions of individual particles can be related to the complex refractive index of their material, then the characterization of a particular ash in terms of its size and composition distributions can lead to the necessary input for car-ing out the calculations, outlined above, to compute radiation transfer in combustion systevis containing that ash.

Thus the key requirement, necessary for the implementation of this approach, is data on the optical constants (i.e. the components $n, k$ of the complex refractive index) as a function of composition, wavelength and temperature covering the range of compositions found in representative ashes. Since, as noted above, and emphasized in texts on the optical properties of particulate matter, it is impractical to extract reliable data on the optical constants of material in particulate form, the only viable approach is to make measurements on homogeneous bulk samples for which well-established techniques are available.

The foregoing arguments provide the rationale for the present program. Characterization of representative flyashes concerning their size and composition distributions constitutes Task 1, while measurements of the optical constants on bulk samples of synthetic slags as a function of relevant ranges of composition, wavelength and temperature constitute Task 2. Task 3 is designed to provide computational capabilities to support the other tasks, while Task 4 is planned to provide an experimental test th the measured optical properties of a dispersion of flyash can indeed be computed reliably from a knowledge of the size and composition distributions of the ash. 


\subsection{Description of Tasks}

\section{TASK 1 - Characterization of Flyash}

Extensive prior analyses of flyash from a wide range of coals plus analyses of the mineral matter in raw coals, together with knowledge of the transformation processes occurring during combustion, lead to the following overall picture of the nature of flyash.

The particle size distribution is very broad with a volume (or mass) mean diameter on the order of $10 \mu \mathrm{m}$. Typically it is well represented by a log normal distribution with the $1 \%$ and $99 \%$ sizes in a cumulative plot by volume occurring at $\sim 1 \mu \mathrm{m}$ and $70 \mu \mathrm{m}$ respectively. Evidence of a distinct submicron fume due to homogeneous condensation of volatile mineral matter is sometimes found, but this fraction can be expected to contribute negligibly to radiation transfer.

With regard to chemical composition, several distinct classes of particle can be identified and plausibly related to their origin and formation mechanisms.

By far the preponderant class, usually representing on the order of $90 \%$ or more of the ash on a mass basis, consists of vitreous (amorphous) material composed primarily of $\mathrm{SiO}_{2}$, $\mathrm{Al}_{2} \mathrm{O}_{3}, \mathrm{CaO}$ and $\mathrm{MgO}$, usually in that order, but containing varying smaller percentages of other metal oxides, notably $\mathrm{Fe}_{2} \mathrm{O}_{3}$. It can appropriately be identified as particles of impure (calcium) aluminosilicate glass derived from the microscopic clay-like mineral inclusions in the coal matrix. As char burnout proceeds these inclusions melt and form liquid globules on the surface of the char (which they do not wet) and are then released into the gas.

These glassy particles tend to be quite spherical with smooth surfaces and of reasonably homogeneous composition as is to be expected from their formation as liquid droplets. As they conl after release from the char surface, they remain in the vitreous state because the cooling rate is much faster than the crystallization rate for the formation of specific phases. The fact that the bulk of most ashes consists of reasonably spherical, homogeneous and vitreous (and therefore optically isotropic) particles is a very fortunate fact, since they satisfy the assumptions of the Mie theory remarkably well.

Micrographs of optically polished sections of ash cast in epoxy resin, shows that these glassy particles sometimes contain a number of small bubbles of gas evolved from the char and trapped in the particles as they form on the char surface. More rarely, large, thin-walled cenospheres are observed which presumably are "glass-blown" when a liquid drop covers a pore in the char from which a relatively large volume of gas is evolved under pressure. Although such cenospheres are very prominent objects in micrographs, their number is usually too small to significantly affect radiation transfer. 
Auger spectroscopic studies of ash often show a thin surface layer composed of volatile metals and high in sulfur (as sulfates) and water. The presence of a thin layer of adsorbed water containing sulfate ions controls the electrical resistance of the surface which is very important in the performance of electrostatic precipitators. However, this surface layer, of different composition from the underlying particle, is too thin $(\leq 100 \AA)$ relative to wavelengths of interest to affect the optical properties of the particle.

Apart from this major class of glassy particles, several distinct minor classes of particle types can be identified, each comprising, at most, a few percent by mass of the flyash. One such class consists of incompletely burned char particles which are clearly identified in optical and SEM micrographs by the fact that they are black, of irregular shape and porous. The mass fraction of char depends on the particular coal and the combustor configuration and operating conditions. In modern combustors the mass fraction of unburned char is normally a few percent at most.

Another minor class consists of particles of adventitious incombustible mineral matter (e.g. quartz) which is contained in the pulverized coal feed. Such particles are usually large and of irregular shape, often showing rounded edges indicating partial melting.

A third minor class consists of magnetite $\left(\mathrm{Fe}_{3} \mathrm{O}_{4}\right)$ which derives from the combustion of pyrite $\left(\mathrm{FeS}_{2}\right)$ particles contained in the coal grind. These magnetite particles are black, generally spherical, magnetic and much denser than the glassy particles. The proportion of magnetite particles depends on the coal type, being largest in high sulfur coals, because the sulfur is mostly associated with pyrite. Recent work has shown that much of the pyrite in the coal grind can be removed by washing/sedimentation with a reduction in $\mathrm{SO}_{\mathbf{x}}$ emissions as high as $50 \%$ in some high sulfur coals.

The ashes selected for characterization are samples from power plants or pilot scale combustors derived from the same seven coals selected for study under the parallel PETC program on "Transformation of Inorganic Coal Constituents in Combustion Systems" which comprise four bituminous, one sub-bituminous and two lignite coals.

A variety of techniques are being used in the characterization of these ashes. The principal method for determining size distributions employs a Coulter Miltisizer which is capable of giving accurate, reliable results of high resolution over a wide dynamic range $\leq 1 \mu \mathrm{m}$ to $\geq 100 \mu \mathrm{m}$.

Automated, computer-controlled, combined SEM/EDX microanalysis will be the principal technique used to determine the distributions of composition and size for a large number $(-1000)$ of particles for each ash. The size distributions will be compared with those obtained by the Coulter counter. 
Other techniques to be used include classification by density using liquids of varying density in a centrifuge, together with classification by size using a wet-sieving method. These techniques can yield density and size separated fractions for further examination by microanalytical techniques such as energy-dispersive X-ray spectroscopy. In addition, the magnetite particles may be separated by magnetic separation. The char content will be determined by low temperature ashing.

\section{TASK 2-Measurements of Optical Constants of Synthetic Slags}

This task is planned to provide the basic optical properties data in a comprehensive and conveniently usable form. The optical constants (i.e., the components of the complex refractive index $m=n-i k$ ) of samples of synthetic slags of controlled compositions will be measured using established techniques involving transmission and surface reflectance methods. The wavelength range will extend from the visible to $12 \mu \mathrm{m}$, and the temperature range will extend to $2000 \mathrm{~K}$.

In earlier work at Stanford, supported by NSF, extensive measurements of this type were made on polished wafers of synthetic slags at temperatures up to $1200 \mathrm{~K}$. A major component of this task will be to extend such measurements to higher temperatures $(\sim 2000 \mathrm{~K})$ where the slag is liquid. This requires the development of modified techniques which present a number of more or less severe technical challenges.

Initially, the optical constants of the basic calcium-aluminosilicate host glass will be determined for the composition range defined by Task 1 . Subsequently, by adding infraredactive mineral oxide constituents in controlled amounts, one at a time, the modifications to $\mathrm{m}(\lambda, \mathrm{T})$ produced by such constituents will be quantitatively determined. The particular constituents (and their range of mass fractions) to be examined will be determined by those disclosed by Task 1, taking account of knowledge of the optical activity at relevant wavelengths of such additions from the literature of glass technology. Specific constituents to be examined will include $\mathrm{Fe}_{2} \mathrm{O}_{3}$, taking especial account of its valence state $\left(\mathrm{Fe}^{2+} / \mathrm{Fe}^{3+}\right.$ ratio), and of $\mathrm{TiO}_{2}$. The contribution of the $\mathrm{OH}$ radical to the optical properties will be evaluated and quantified if significant.

The experimental data on $m(\lambda, T)$ as a function of composition, over the range relevant to coal ashes, will be reduced to generate simple correlation formulae. The latter will constitute the data base necessary to calculate the radiative properties of bulk slags and ash dispersions required for understanding and computing radiative transfer in coal combustion systems. 


\section{TASK 3 - Sample Calculations of the Radiant Properties of Flyash Dispersions}

This task is intended to provide computational capabilities to support the other tasks. It includes the following components.

(i) A Mie scattering code to calculate the absorption and scattering efficiencies and phase function of a single sphere of specified size parameter and complex refractive index. A modified Mie code will also allow such computations for hollow spheres.

(ii) A code to convolve the results from (i) over a specified size distribution and loading to compute the absorption and scattering coefficients and phase function of a homogeneous polydispersion.

(iii) A code to sum the results of (ii) for a number of classes of particles of varying refractive indices and size distributions, i.e. for a heterogeneous polydispersion.

(iv) A radiation transfer code to calculate the absorption, scattering and emission characteristics of a homogenous, isothermal slab of dispersed ash on a spectral basis.

(v) A code to integrate the spectral results from (iv) over wavelength to yield the total radiative properties of the slab.

Calculations using these codes will be used to provide sensitivity analyses to guide the characterization work of Task 1, and to design and evaluate the results of Task 4.

\section{TASK 4 - Measurement of the Radiative Properties of Flyash Dispersions}

The purpose of this laboratory scale experiment is to test our ability to pre 1 ict the measured radiative properties of a dispersion of well-characterized flyash. As such it will provide a critical test of the effectiveness of the overall approach adopted in this program. 


\subsection{PROGRESS IN THE PAST QUARTER}

\subsection{Task 1: Characterization of Fly Ash}

As described later in the section on Task 4 , it is necessary to obtain quantitative information about the cenospheric content of the ashes, as well as densities of individual ash particles in order to explain the results of the transmittance measurements. The latter is needed to determine the refractive indices of different compositional classes of ash and thus account for ash heterogeneity. The following sections discuss the data obtained in these two areas in the last quarter.

\subsubsection{Densities of Individual Fly Ash Particles}

The density of slags, computed by linear combination of the densities of the component oxides (in crystalline or vitrified state) in proportion to their composition, systematically overpredicts the measured density. This over-prediction probably occurs because the atoms are packed more loosely in the multi-component glass (which is essentially a super-cooled liquid) compared to the individual oxides. By measuring the densities of glasses prepared with different proportions of constituent oxides, one can calculate an effective density $\left(\rho_{e}\right)$ of each oxide in the glass. This effective density is not unique, i.e., the effective density of $\mathrm{SiO}_{2}$ in $\mathrm{SiO}_{2}-\mathrm{CaO}-\mathrm{Na}_{2} \mathrm{O}$ glass is slightly different from the effective density of $\mathrm{SiO}_{2}$ in $\mathrm{SiO}_{2}-\mathrm{K}_{2} \mathrm{O}-\mathrm{Na}_{2} \mathrm{O}$ glass. Table 1 below shows the effective densities as determined by Appen [1]. The density of a slag of specified composiiton can be computed by adding the fractional density contributions of each oxide.

The effective density is higher than that of the pure oxides in the cases $\mathrm{SiO}_{2}, \mathrm{CaO}$, $\mathrm{Na}_{2} \mathrm{O}, \mathrm{K}_{2} \mathrm{O}$, and $\mathrm{BaO}$. The effective density of iron oxide is not available. However, using the available effective densities, it is possible to obtain an effective density of iron oxide so that the error is minimized. The average r.m.s. error, $E$, is computed from the calculated density $\left(\rho_{c}\right)$ and the measured density $\left(\rho_{m}\right)$ of each of $N$ slags as follows:

$$
E=\sqrt{\frac{\sum_{i=1}^{N}\left[\left(\rho_{m, i}-\rho_{c, i}\right) / \rho_{m, i}\right]^{2}}{N}}
$$

Here, $\rho_{c}=\sum_{j=1}^{9} X_{j} \rho_{e, j}, X_{j}$ being the normalized mass fraction of the $j^{\text {th }}$ oxide. 
Table 1: Densities of Oxides Present in Fly Ashes

\begin{tabular}{|c|c|c|}
\hline Oxide & Density $\left(\rho_{o}\right)$, (State) & Effective Density $\left(\rho_{e}\right)$ \\
\hline $\mathrm{SiO}_{2}$ & 2.20 (Fused silica) & 2.28 \\
\hline $\mathrm{Al}_{2} \mathrm{O}_{3}$ & 3.99 (Corundum) & 2.5 \\
\hline $\mathrm{CaO}$ & 3.32 (Lime) & 3.9 \\
\hline $\mathrm{MgO}$ & 3.65 (Periclare) & 3.3 \\
\hline $\mathrm{Na}_{2} \mathrm{O}$ & 2.27 & 3.1 \\
\hline $\mathrm{K}_{2} \mathrm{O}$ & 2.32 & 2.8 \\
\hline $\mathrm{BaO}$ & 5.72 & 7.0 \\
\hline $\mathrm{TiO}_{2}$ & 3.84 & 3.8 \\
\hline
\end{tabular}

Table 2: Calculation of Oxide Densities

\begin{tabular}{|c|c|c|c|c|c|}
\hline Slag & $\rho_{m}$ & $\rho_{c}$ & $\%$ Diff. & Using $\rho_{o}$ & $\%$ Diff. \\
\hline Upper Freeport & 2.67 & 2.61 & -2.15 & 3.14 & 17.78 \\
\hline Kentucky \# 9 & 2.80 & 2.65 & -5.47 & 3.18 & 13.71 \\
\hline Illinois \# 6 & 2.69 & 2.77 & 2.93 & 3.27 & 21.64 \\
\hline Beulah & 2.98 & 2.82 & -5.44 & 3.45 & 15.71 \\
\hline Beulah (Cyclone) & 2.92 & 2.77 & -5.20 & 3.40 & 16.39 \\
\hline San Miguel & 2.39 & 2.48 & 3.79 & 2.74 & 14.92 \\
\hline Eagle Butte & 2.89 & 3.03 & 4.77 & 3.31 & 14.83 \\
\hline SA00 & 2.54 & 2.52 & -0.66 & 2.88 & 13.24 \\
\hline SA05 & 2.60 & 2.58 & -0.69 & 2.98 & 14.78 \\
\hline SA10 & 2.64 & 2.65 & 0.21 & 3.10 & 17.40 \\
\hline SA20 & 2.72 & 2.75 & 1.22 & 3.29 & 21.04 \\
\hline
\end{tabular}

The effective densities of the primary constituent oxides, $\mathrm{SiO}_{2}, \mathrm{Al}_{2} \mathrm{O}_{3}, \mathrm{CaO}$, and $\mathrm{Fe}_{2} \mathrm{O}_{3}$ affect $\rho_{c}$ most. Calculations showed that a lower $\rho_{e}$ for $\mathrm{CaO}$ gave better predictions of slag densities. By trial and error, it was determined that $\rho_{e}$ of 3.4 and 3.8 for $\mathrm{CaO}$ 
and $\mathrm{Fe}_{2} \mathrm{O}_{3}$, respectively, minimized $E$ to $3.56 \%$. It is reiterated that these values of $\rho_{e}$ are not unique, but are engineering approximatations for the complex physics of glasses. Nevertheless, they predict the densities of the slags quite well as can be seen in Table 2 . The data for the four synthetic SAXX slags are taken from Goodwin [2]. Note that $E=16.71 \%$ when actual oxide densities, $\rho_{o}$, are used instead of $\rho_{e}$. The quantity $\%$ Diff.' in the column is defined as $\left(\rho_{m}-\rho_{e}\right) / \rho_{m}$.

As outlined in the QPR of May, 1991, the density of an ash particle, $\rho_{e}$, is needed to determine its spectral complex refractive index. In the absence of a database for computing more accurate densities of glassy ash particles, the values of $\rho_{e}$ presented above are being used. Because $n \sim \sqrt{\rho_{c}}$, the error in $n$ is smaller than that in $\rho_{c}$.

\subsubsection{Cenospheric Content of the Ashes}

Among the major constituents of any ash particle, the one with the lowest density is silica (density $=2.2 \mathrm{~g} / \mathrm{cc}$ ). CCSEM results showed that there are few ash particles that are pure silica. Because the density of the next major constituent, alumina, is significantly higher, it is reasonable to conclude that all ash of density $<2.4 \mathrm{~g} / \mathrm{cc}$ are, to varying degrees, cenospheric. Centrifugal separation was carried out on the six ashes to ditermine their cenospheric content. The results are shown in Table 3. It is seen that San Miguel is almost entirely cenospheric while the calcia rich Eagle Butte has very few cenospheres by mass. The three bituminous ashes have cenospheric contents varying between $14 \%$ and $24 \%$.

The size distributions of both density fractions were measured with the Coulter Multisizer for all the ashes except the San Miguel ash. (Sufficient amount of the denser fraction of the San Miguel ash was not available). Figures 1--10 show the size distributions in both cumulative and differential form on the bases of numbur, area, and volume. It is seen that $D_{50 \%}$ of the cenospheric fraction of an ash is two to three times larger than $D_{50 \%}$ of the fraction with $\rho>2.2 \mathrm{~g} / \mathrm{cc}$. It appears that most of the size distributions (especially the volume distributions) can be described well by log-normal functions. This observation is consistent with the size distributions of six density classes 
that were reported in the QPR of May, 1991.

Table 3: Ash Mass Fraction with Density $<2.2 \mathrm{~g} / \mathrm{cc}$.

\begin{tabular}{|c|c|}
\hline Fly Ash & Mass \% with $\rho<\mathbf{2 . 2} \mathbf{g} / \mathbf{c c}$ \\
\hline Upper Freeport (Bituminous) & $23.9 \%$ \\
\hline Kentucky \# 9 (Bituminous) & $19.3 \%$ \\
\hline Illinois \# 6 (Bituminous) & $14.6 \%$ \\
\hline Eagle Butte (Sub-bituminous) & $3.6 \%$ \\
\hline Beulah (Lignite) & $7.7 \%$ \\
\hline San Miguel (Lignite) & $96.9 \%$ \\
\hline
\end{tabular}

It is possible that the small particles that cause some of the cenospheric distributions to be bimodal (e.g., Figures 1 and 9) may not be cenospheres. As discussed in the QPR dated May, 1991, some of the smaller non-cenospheric ash particles are carried over into the lighter fraction during centrifugal separation. This systematic error leads to an overestimation of the cenospheric content and an under-estimation of $D_{v}$. However, if the densities of the two separated fractions are known, and a cenospheric cut-off diameter (i.e., ash particles below the cut-off diameter do not contain bubbles) is determined, a correction can be incorporated. SEM study of the density separated fractions are currently being conducted, and results of further analysis, along with parameters of the best-fit log-normal functions for the size distributions in this section will be reported in the next QPR. 

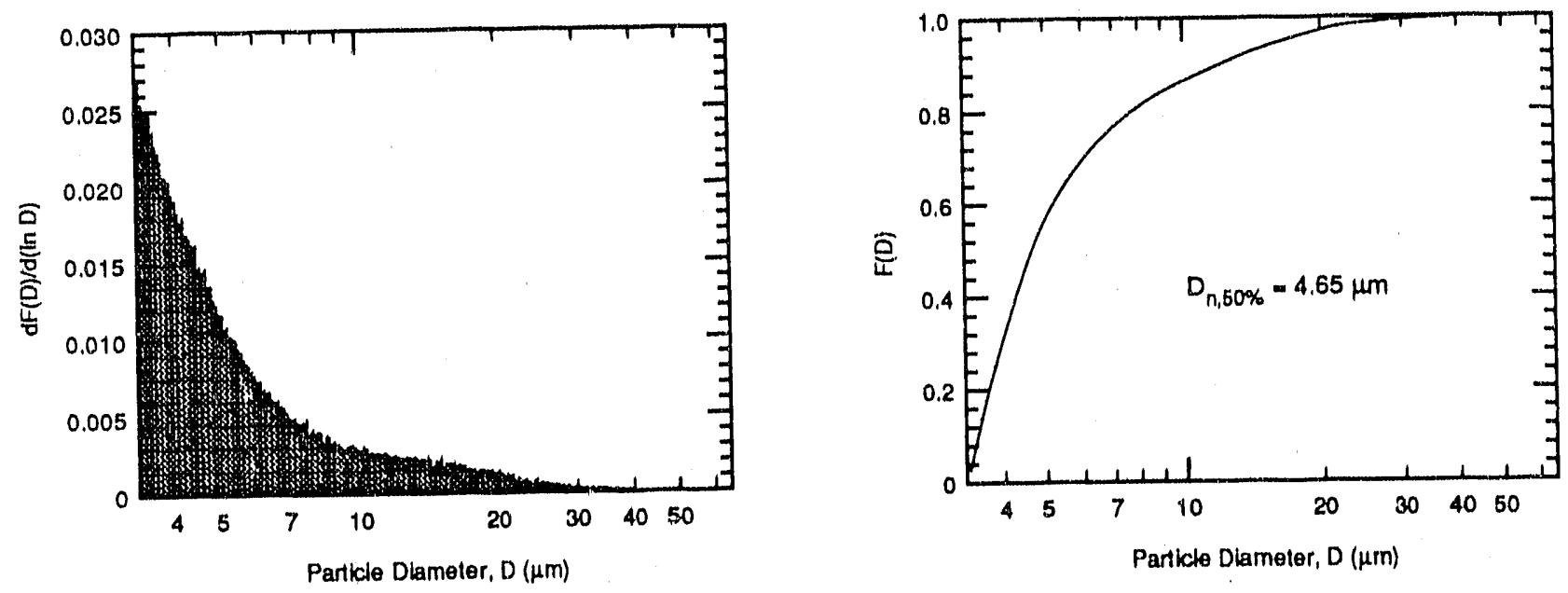

Number/Count Distribution
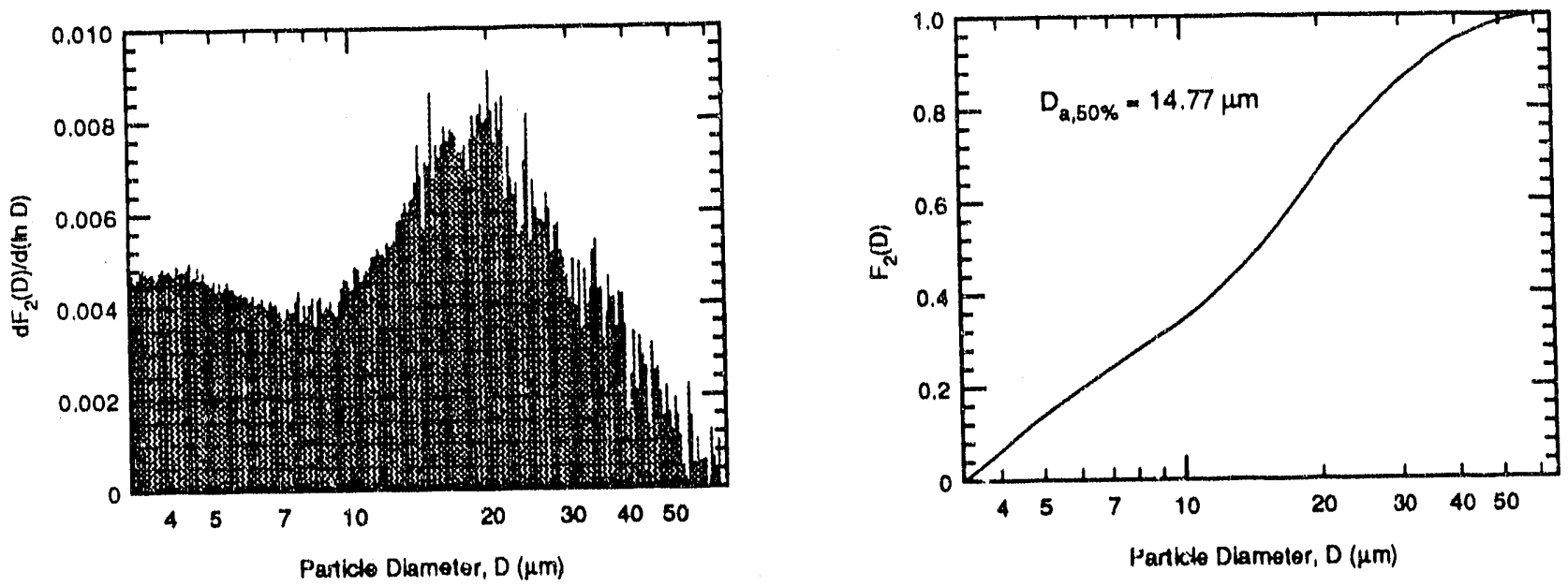

Area Distribution
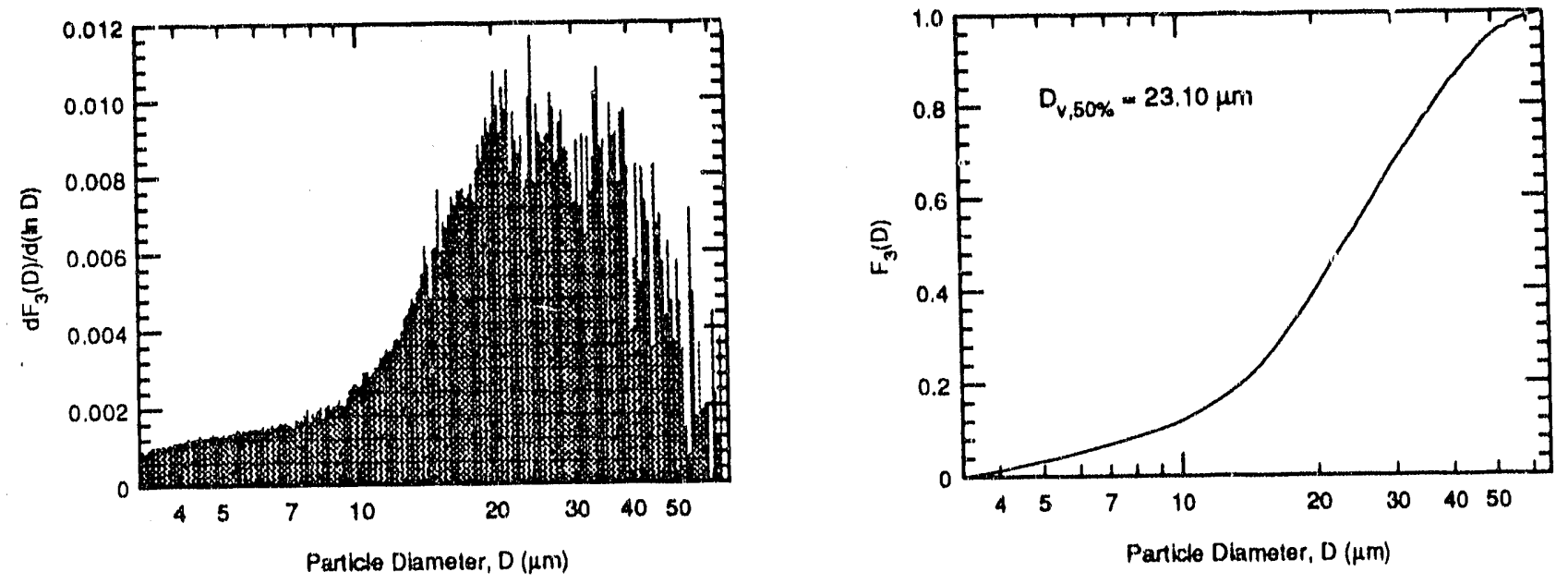

Volume Distribution

Figure 1: Differential and Cumulative Size Distributions for Upper Freeport Baghouse Ash, Density <2.2 g/cc. Multisizer Orifice Diameter $=100 \mu \mathrm{m}$. Total Particle Count $=178,595$ 

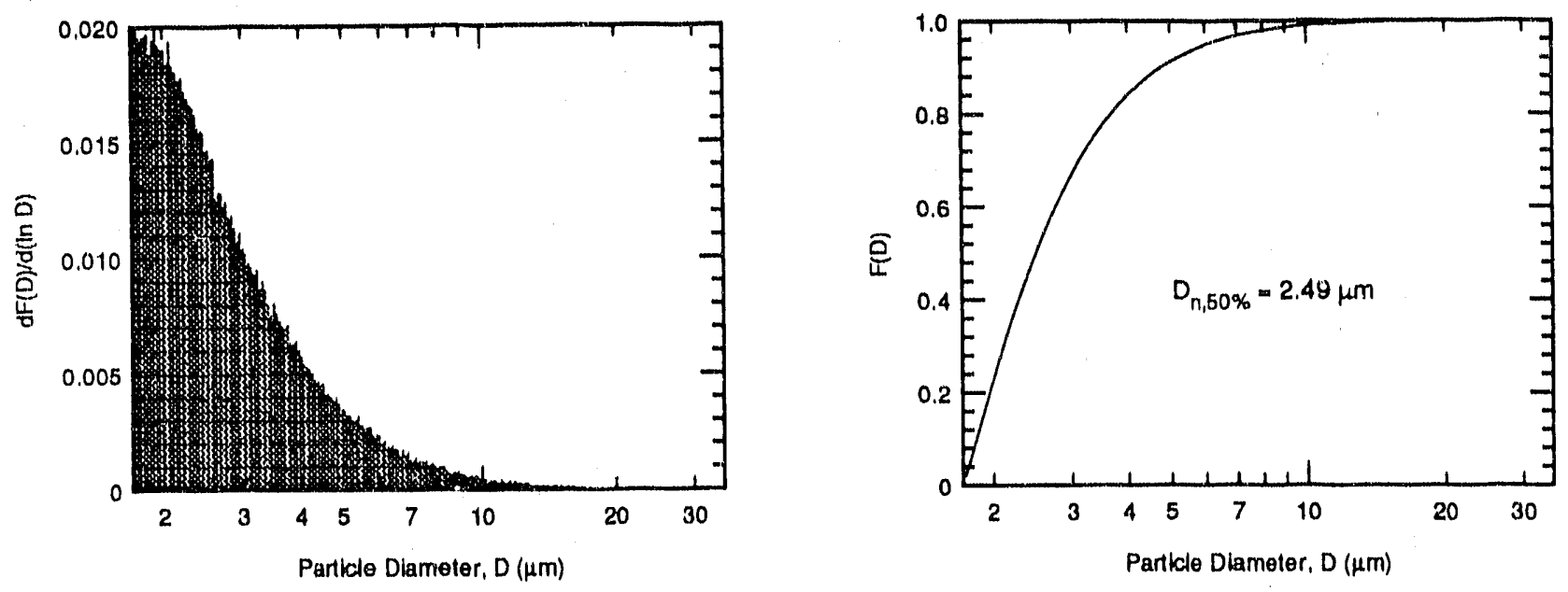

Number/Count Distribution
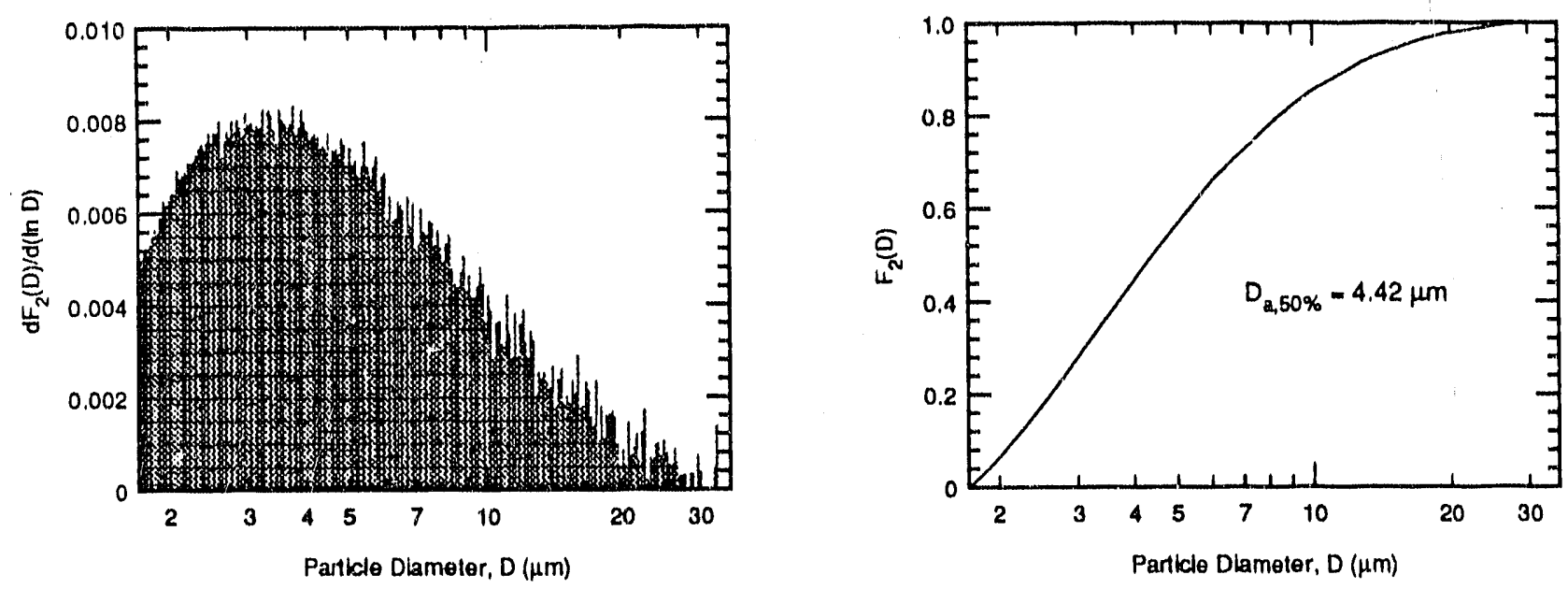

Area Distribution
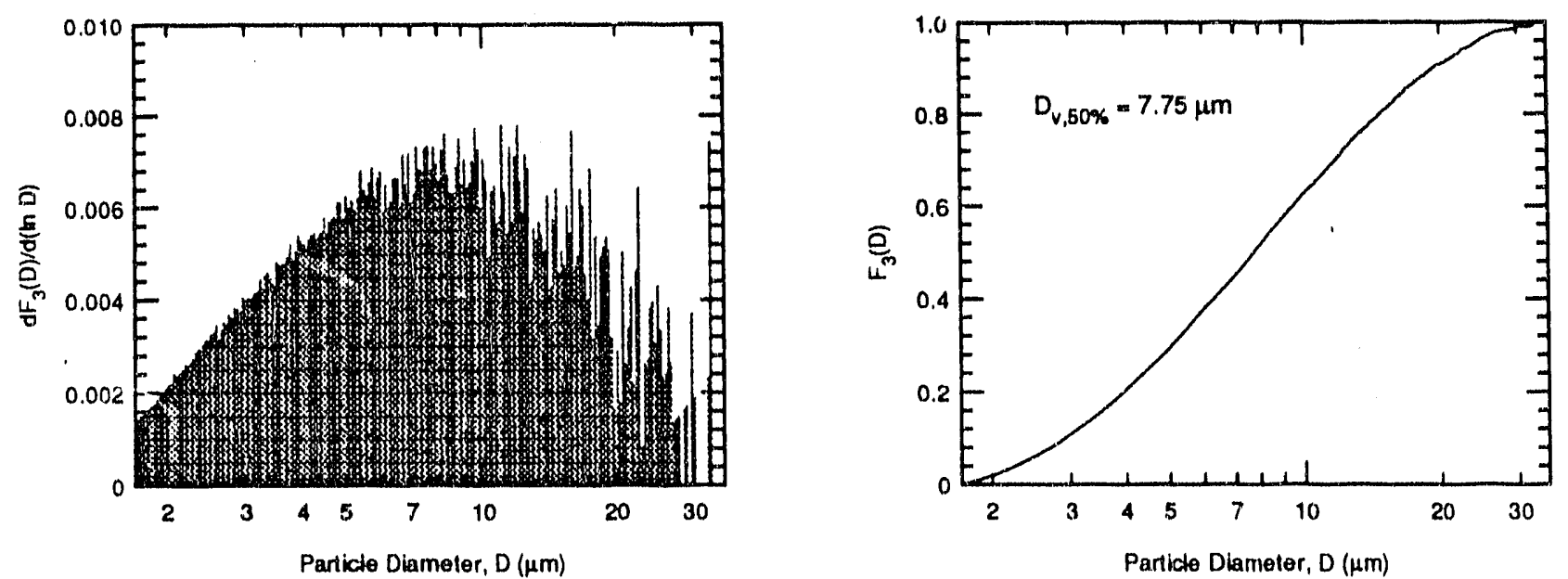

Volume Distribution

Figure 2: Differential and Cumulative Size Distributions for Upper Freeport Ash, Density $>2.2 \mathrm{~g} / \mathrm{cc}$. Multisizer Orifice Diameter $=50 \mu \mathrm{m}$. Particle Count $=197,375$ 

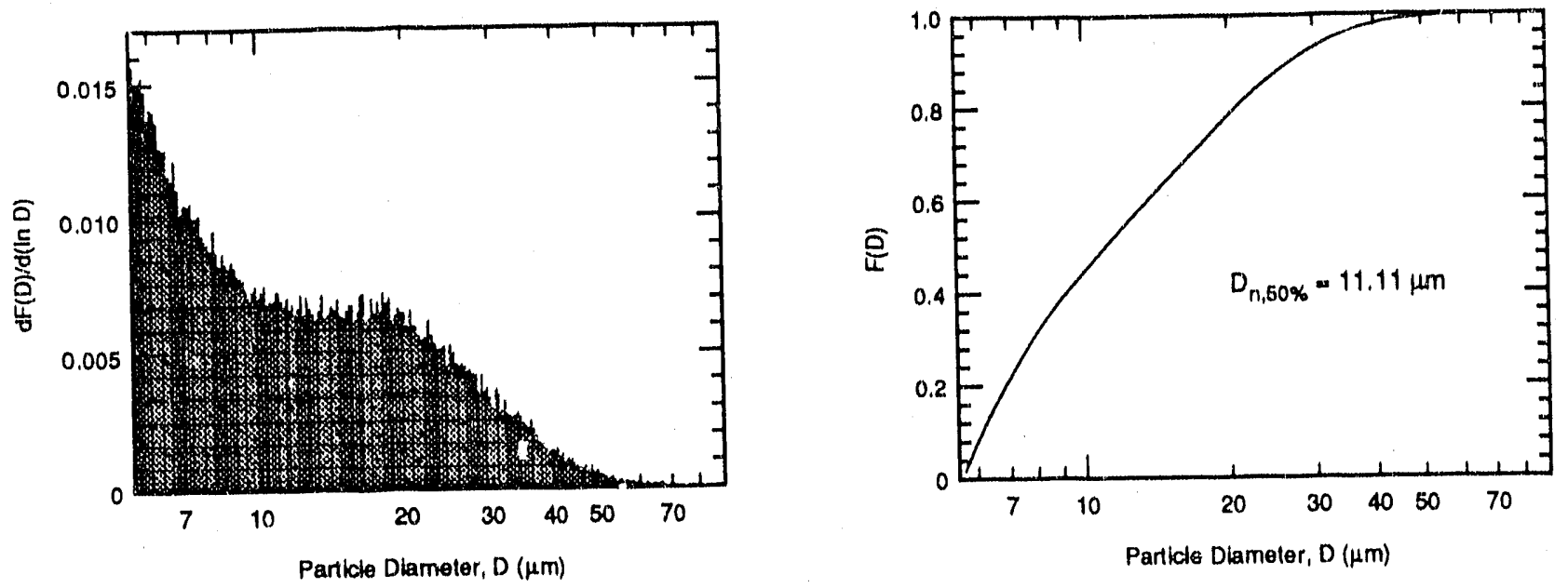

Number/Count Distribution
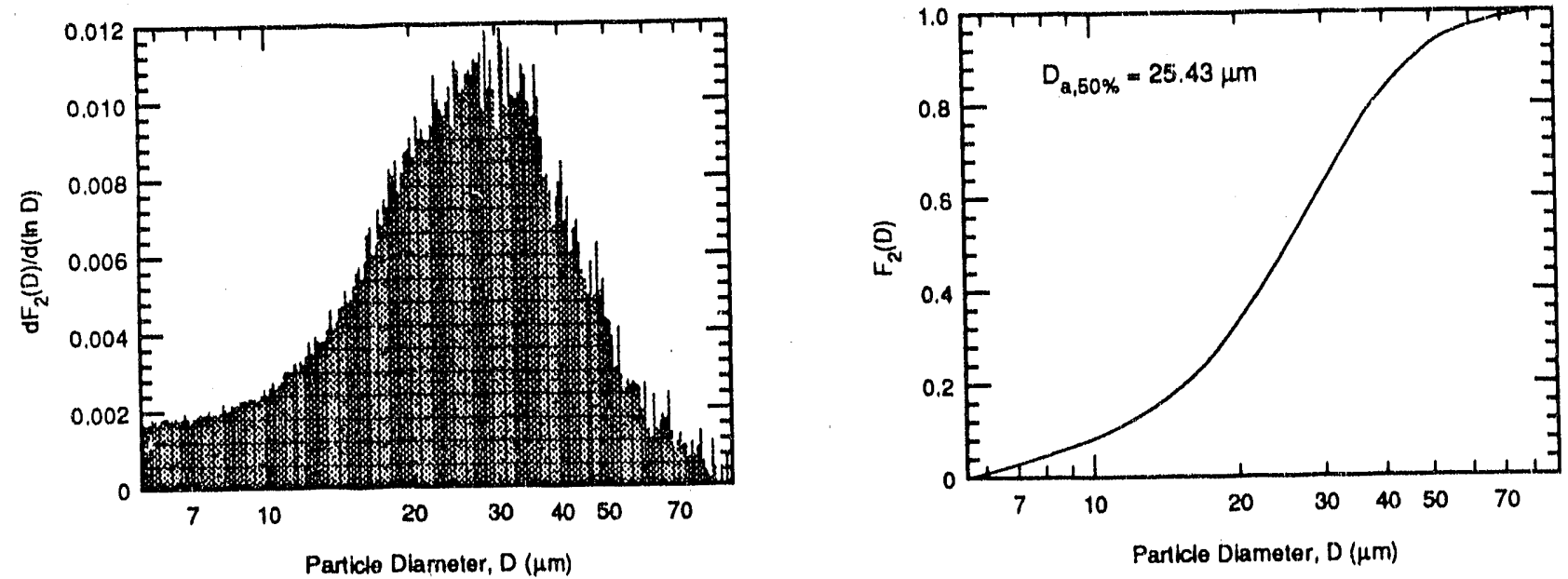

Area Distribution
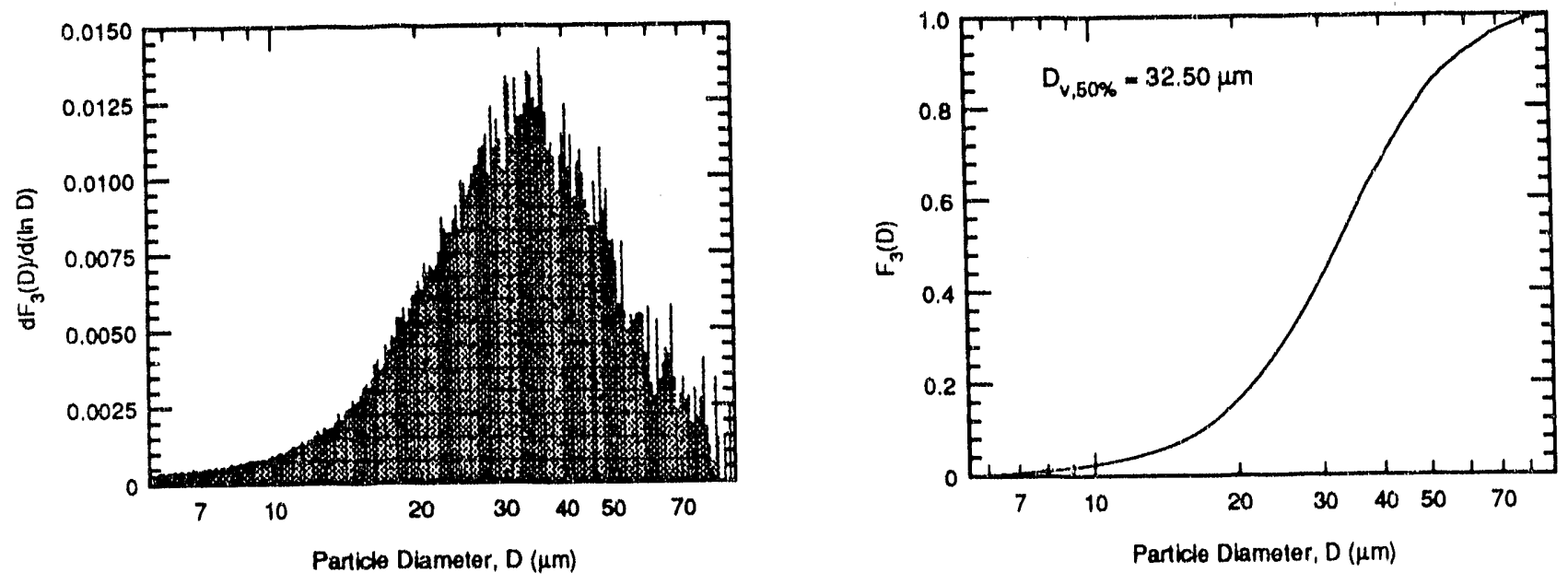

Volume Distribution

Figure 3: Differential and Cumulative Size Distributions for Illinois \#6 Baghouse Ash, Density < $2.2 \mathrm{~g} / \mathrm{cc}$. Multisizer Orifice Diameter $=140 \mu \mathrm{m}$. Total Particle Count $=103,730$. 

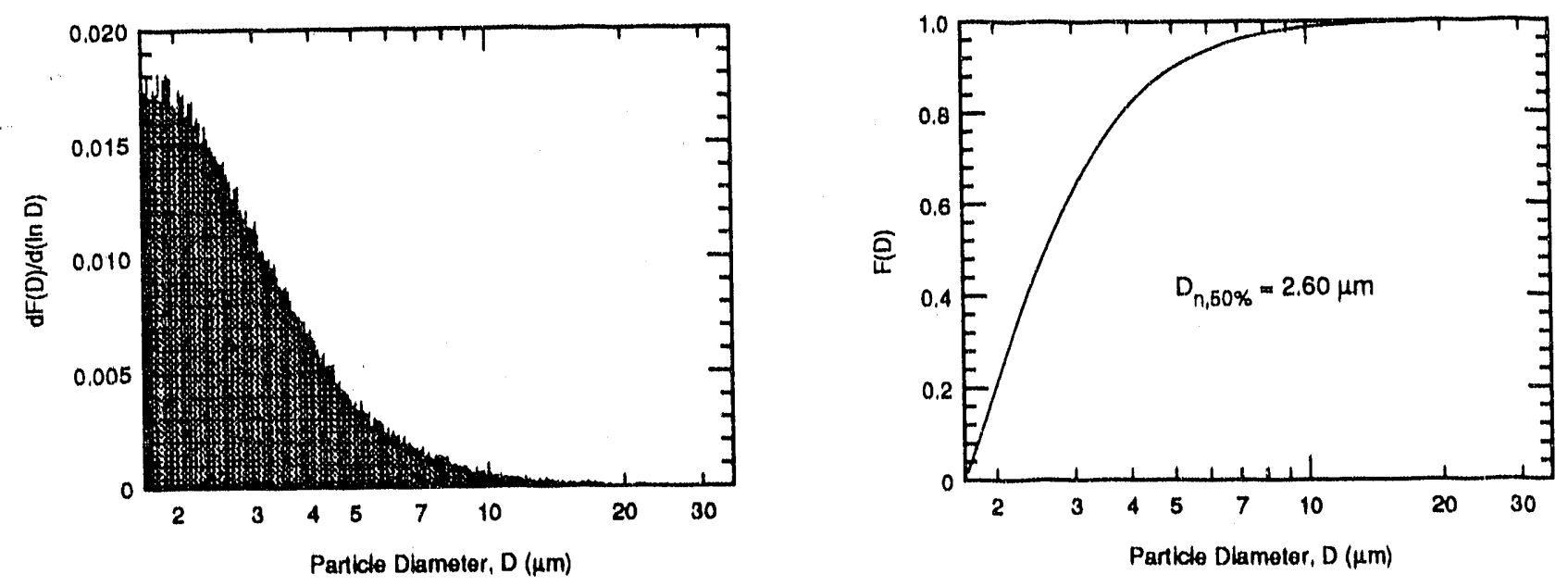

Number/Count Distribution
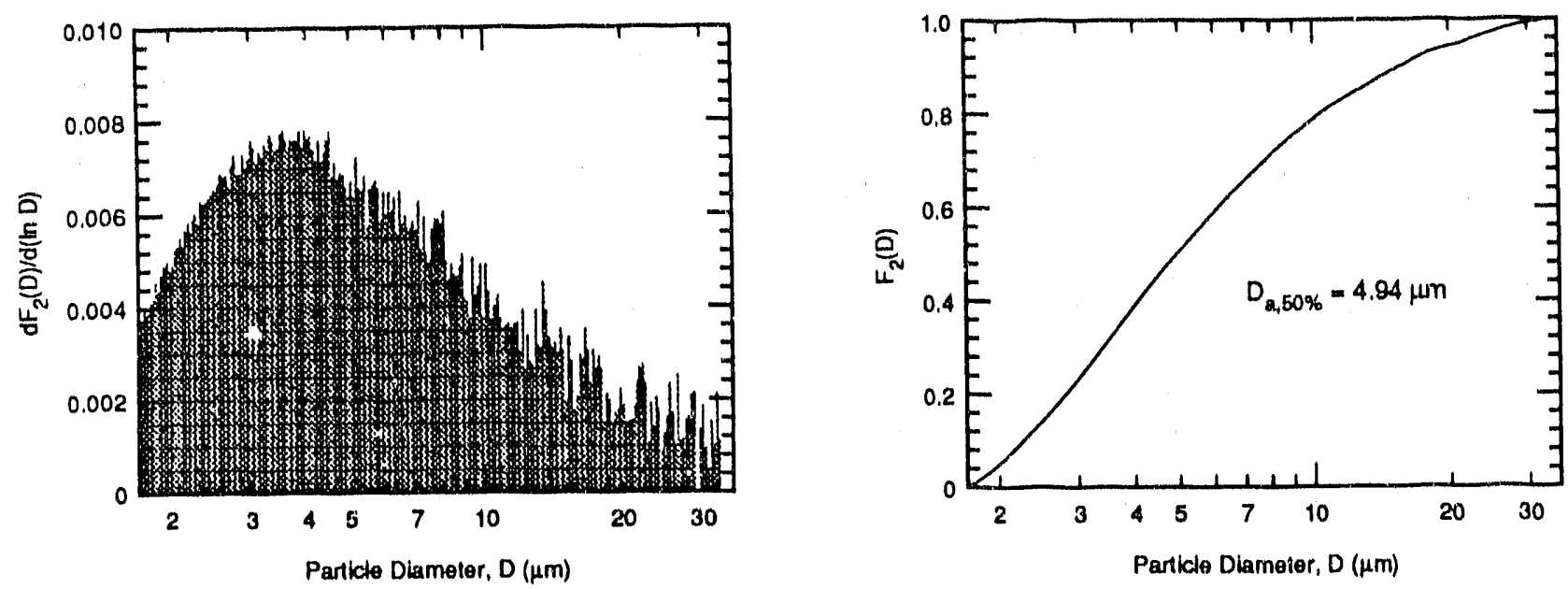

Area Distribution
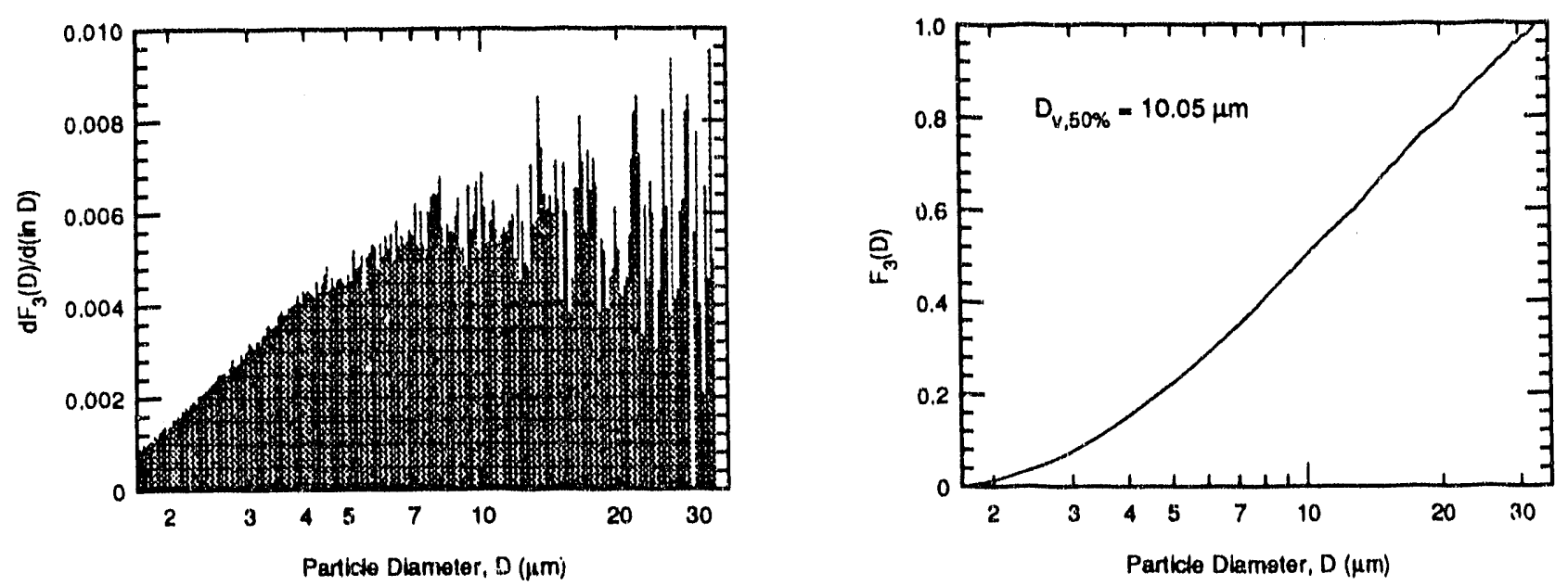

Volume Distribution

Figure 4: Differential and Cumulative Size Distributions for llinois \#6 Ash, Density > $2.2 \mathrm{~g} / \mathrm{cc}$.

Multisizer Orifice Diameter $=50 \mu \mathrm{m}$. Particle Count $=141,540$ 

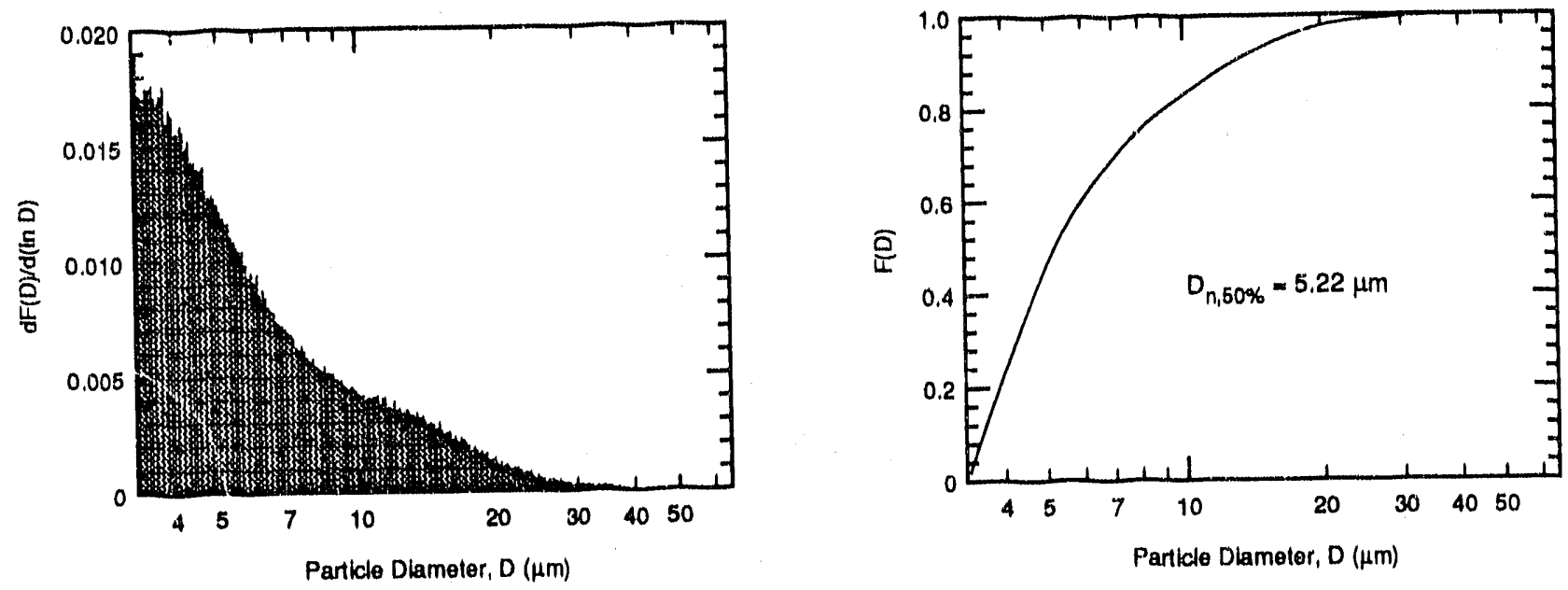

Number/Count Distribution
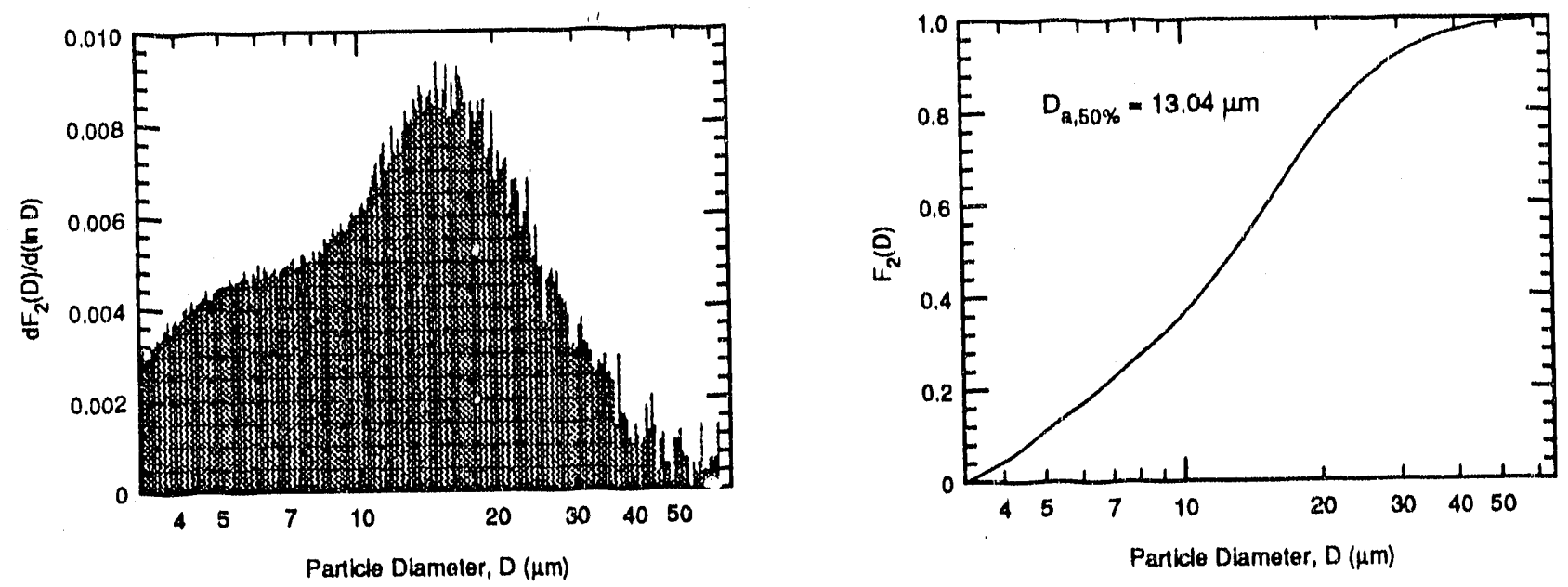

Area Distribution
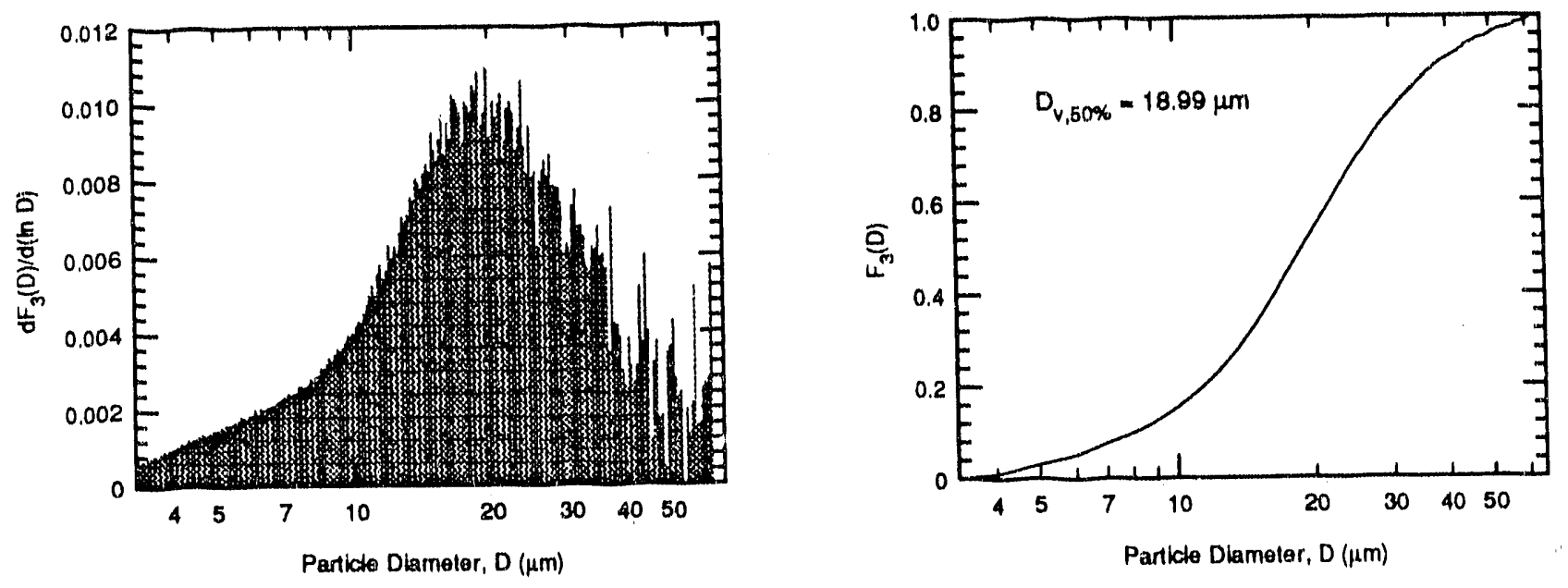

Volume Distribution

Figure 5: Differential and Cumulative Size Distributions for Kentucky \#9 Baghouse Ash, Density <2.2 g/c.c. Multisizer Orifice Diameter $=100 \mu \mathrm{m}$. Total Particle Count $=231,345$ 

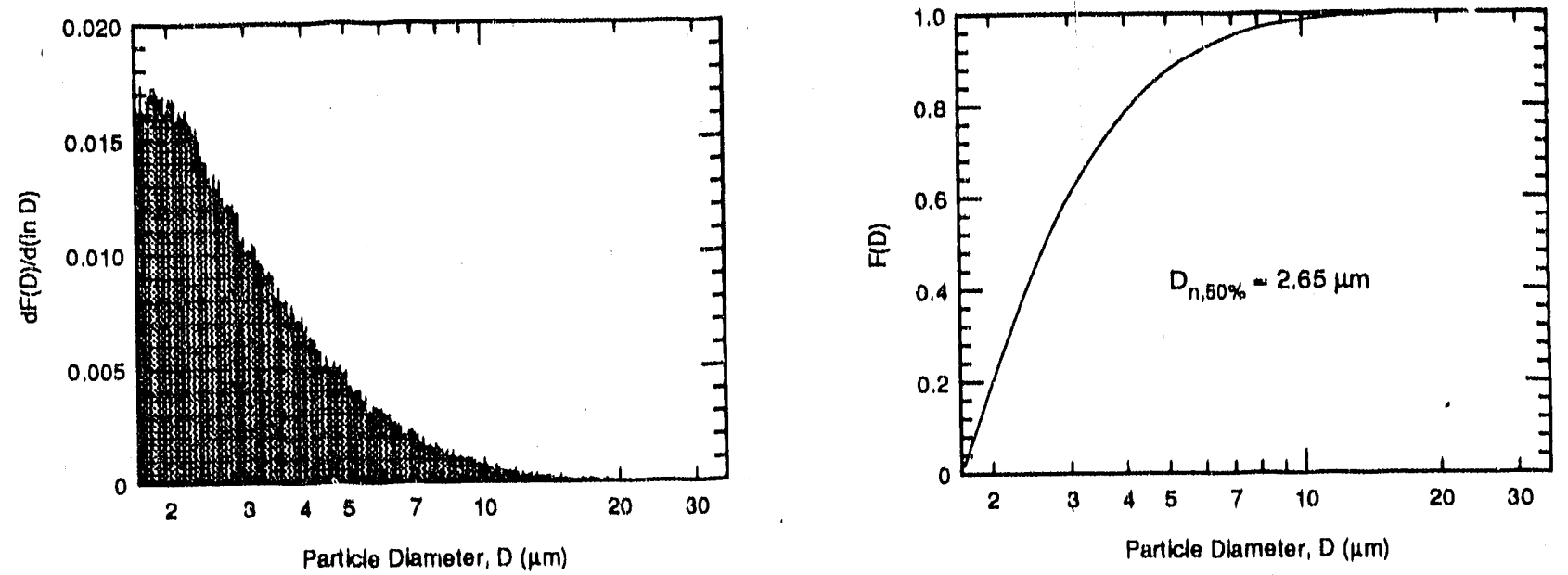

Number/Count Distribution
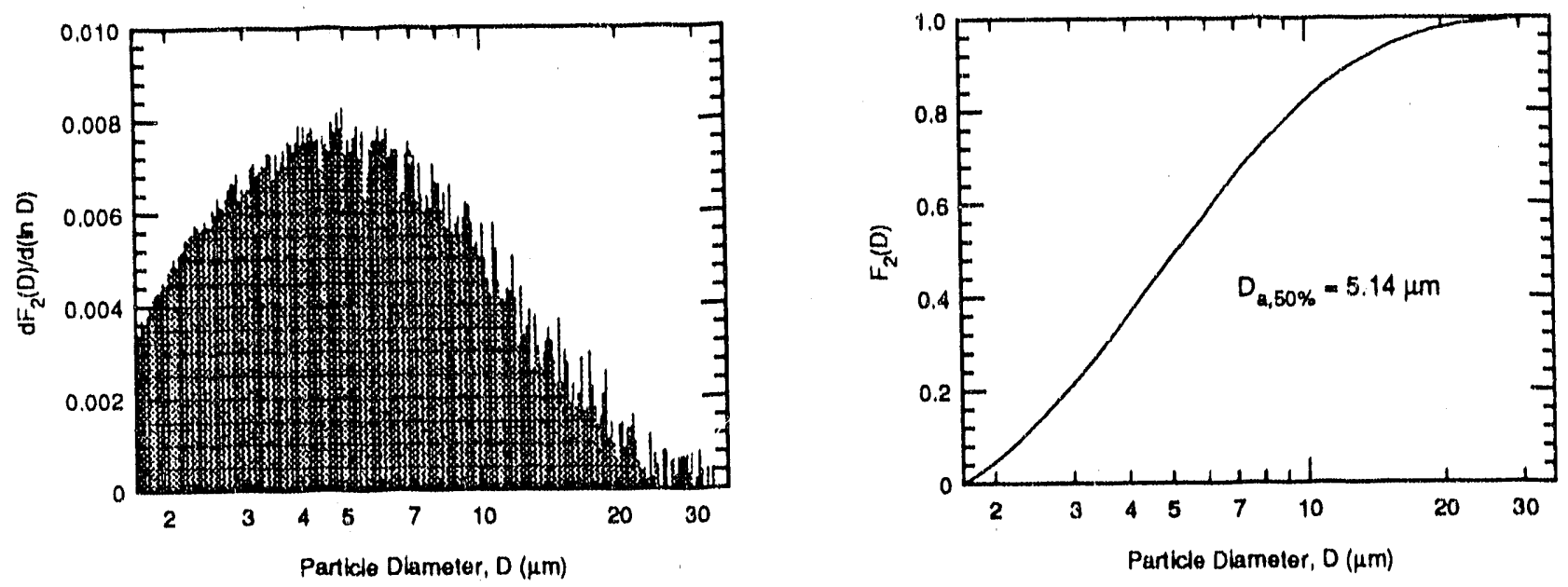

Area Distribution
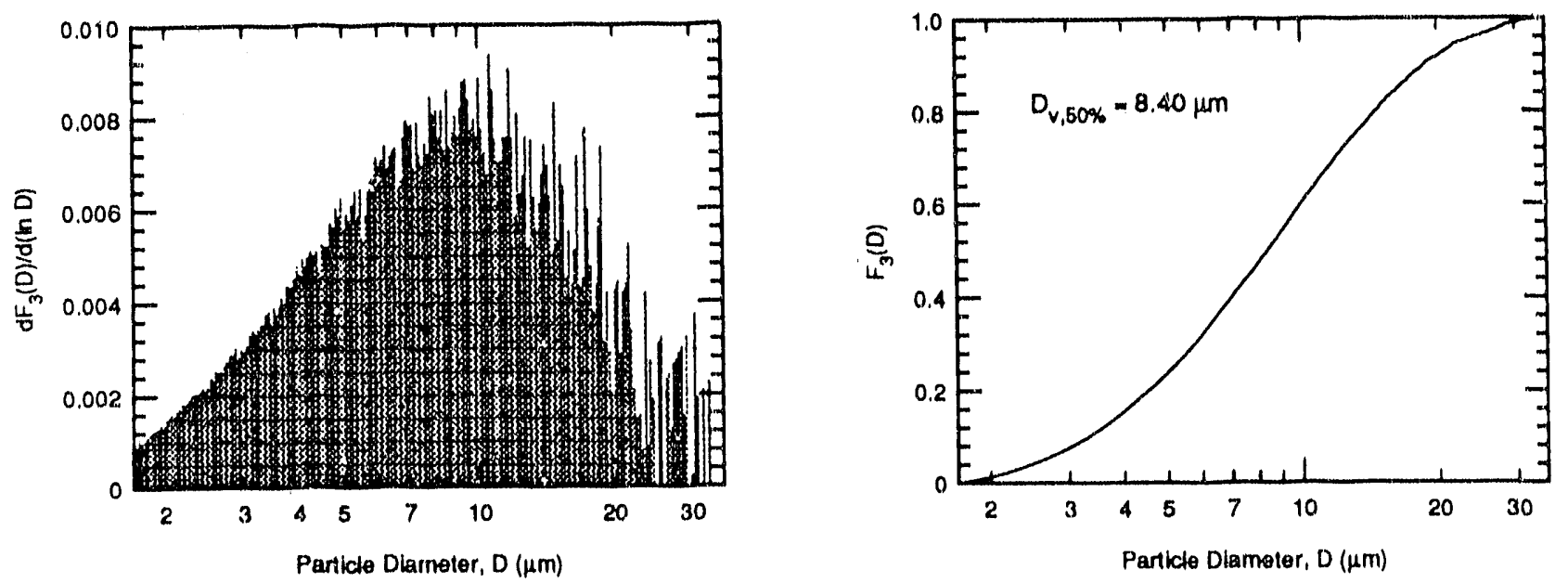

Volume Distribution

Figure 6: Differential and Cumulative Size Distributions for Kentucky \#9 Ash, Density > $2.2 \mathrm{~g} / \mathrm{cc}$. Multisizer Orifice Diameter $=50 \mu \mathrm{m}$. Particle Count $=162,457$ 

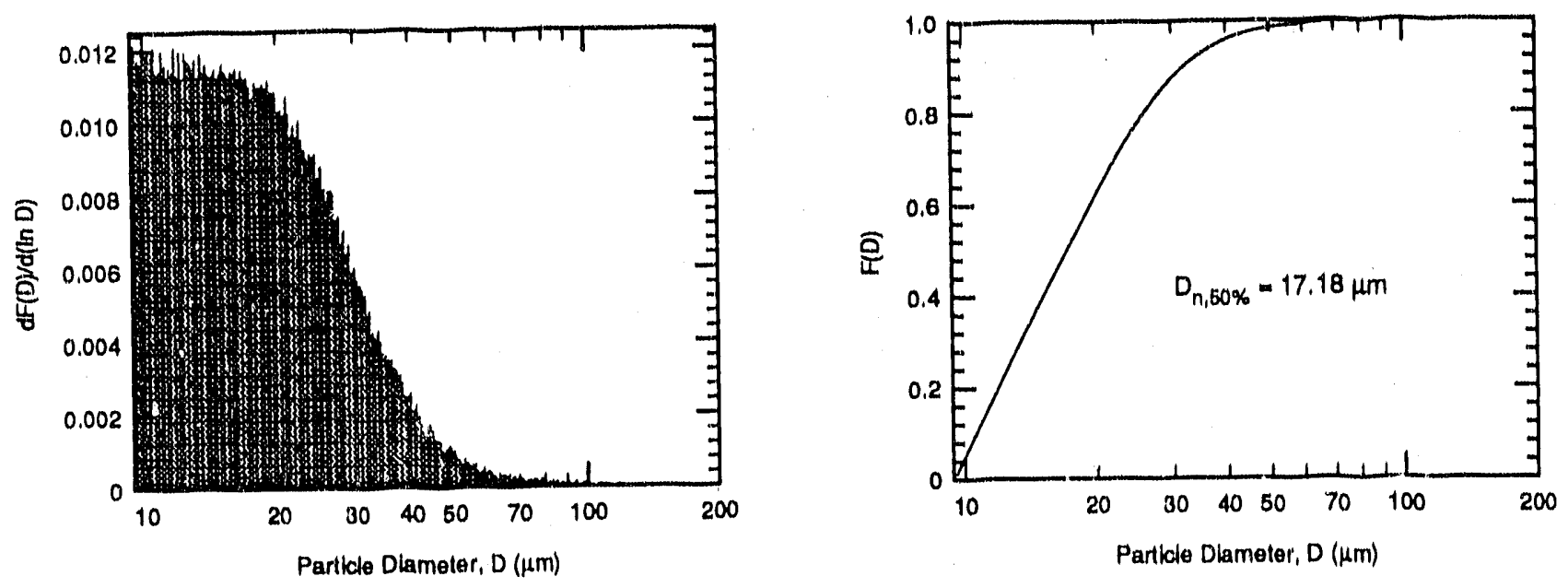

Number/Count Distribution
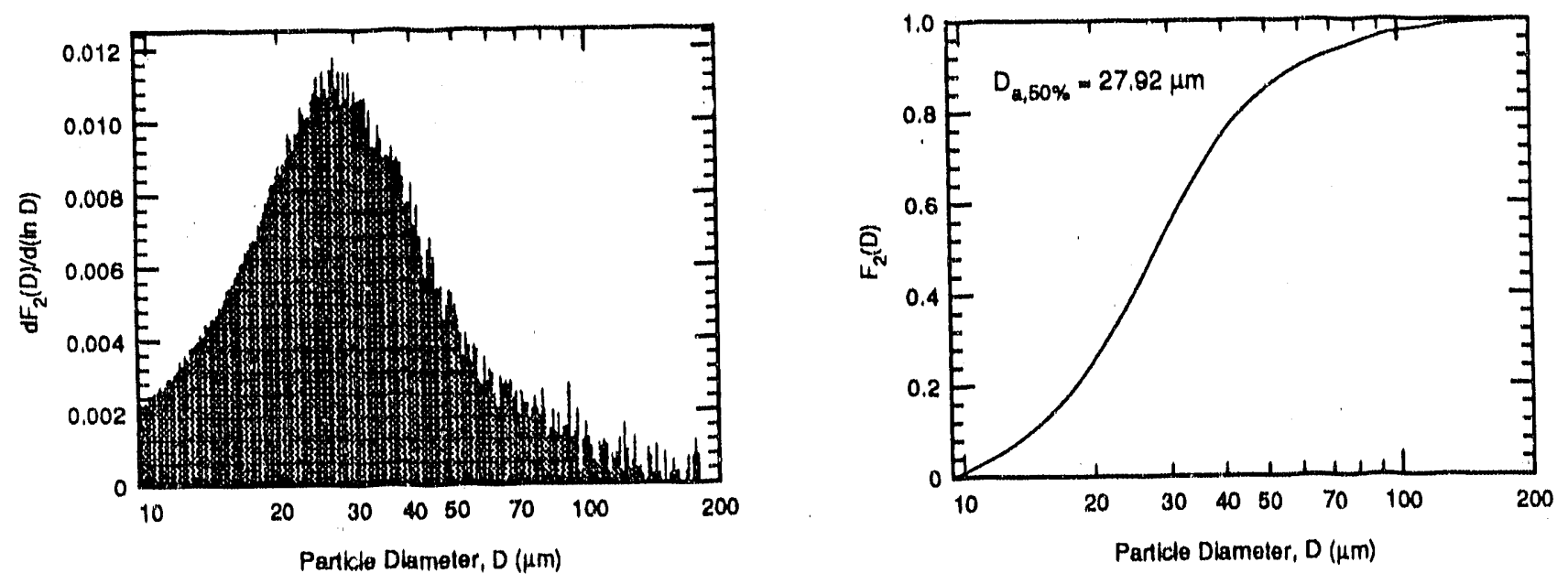

Area Distribution
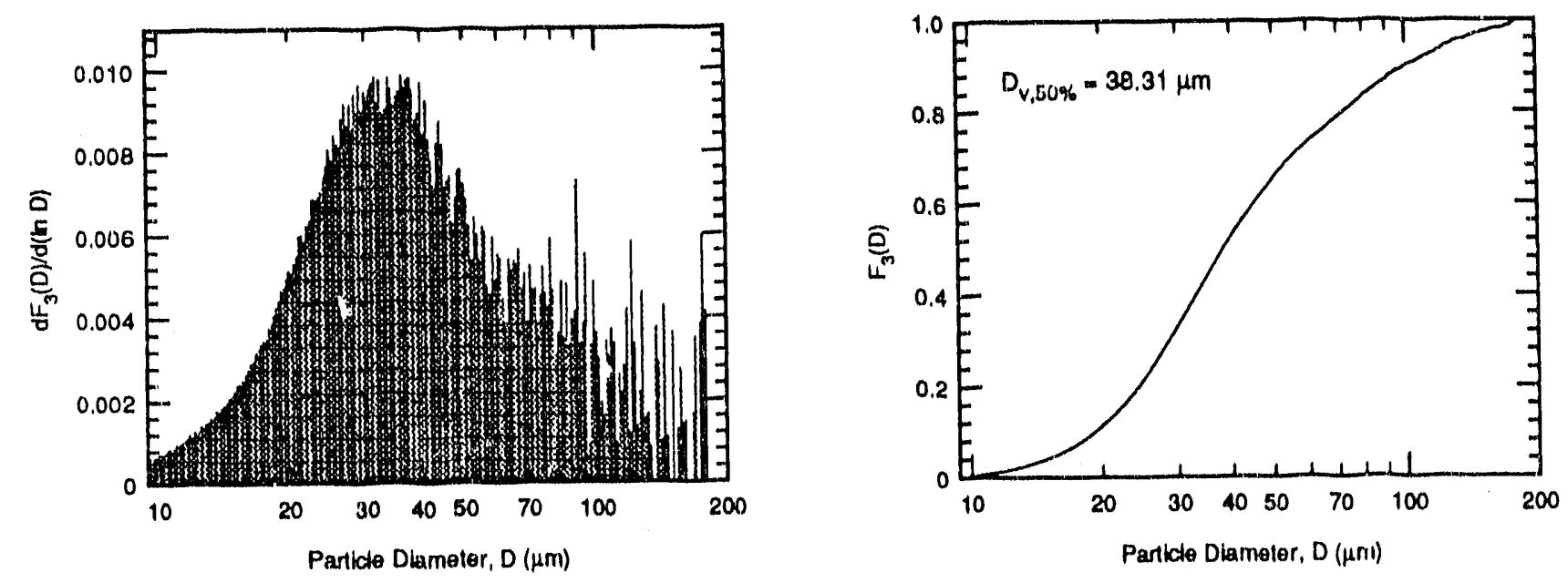

Volume Distribution

Figure 7: Differential and Cumulative Size Distributions for Eagle Butte Ash, Density $<2.2 \mathrm{~g} / \mathrm{cc}$. Multisizer Orifice Diameter $=280 \mu \mathrm{m}$. Total Particle Count $=163,007$ 

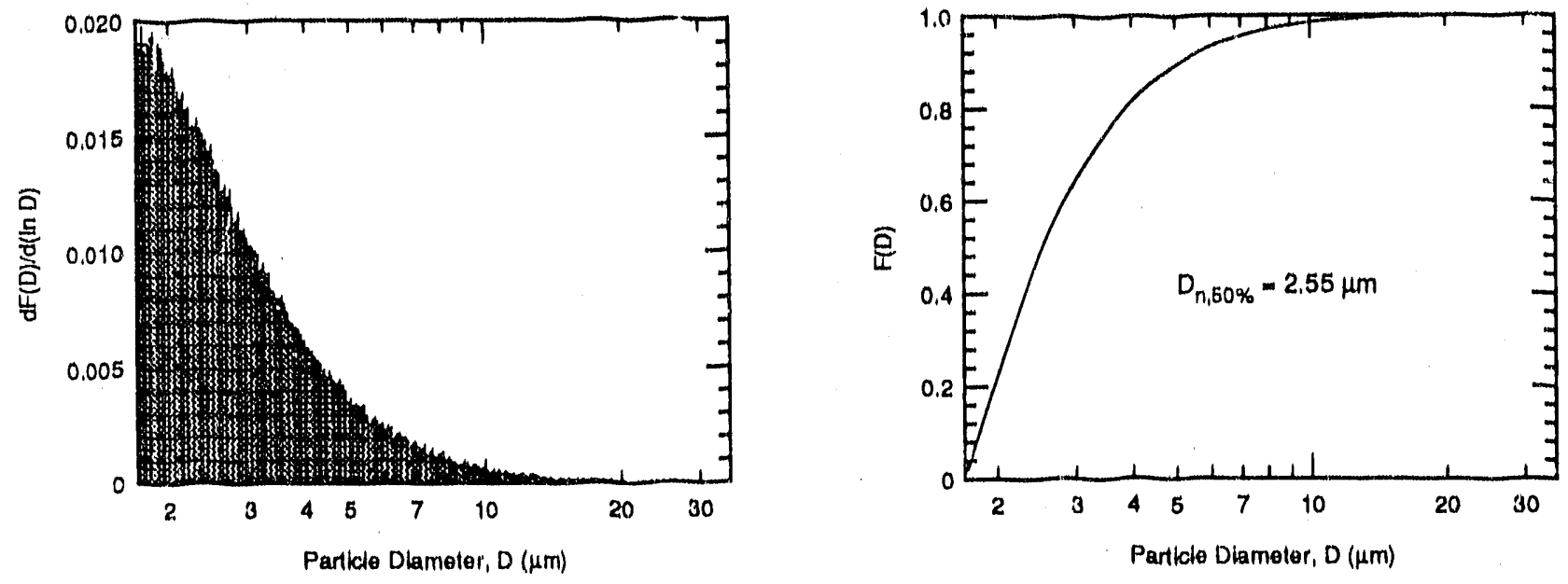

Number/Count Distribution
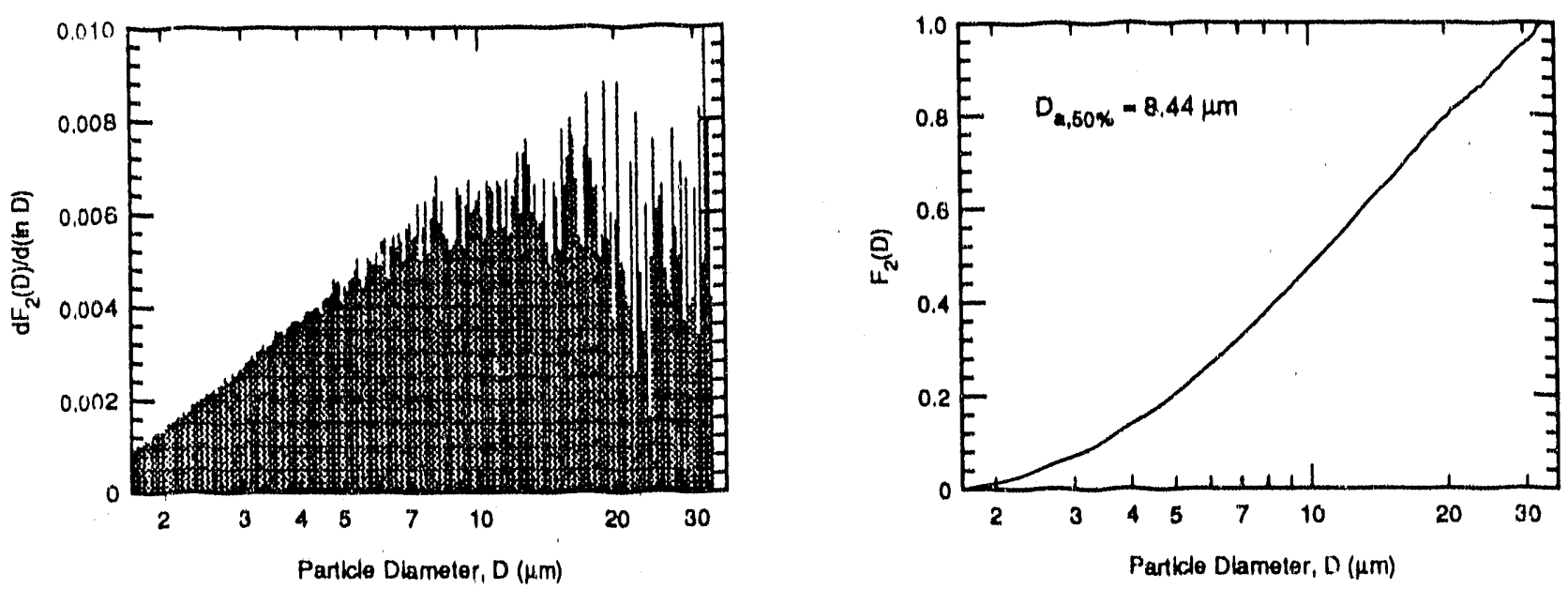

Area Distribution
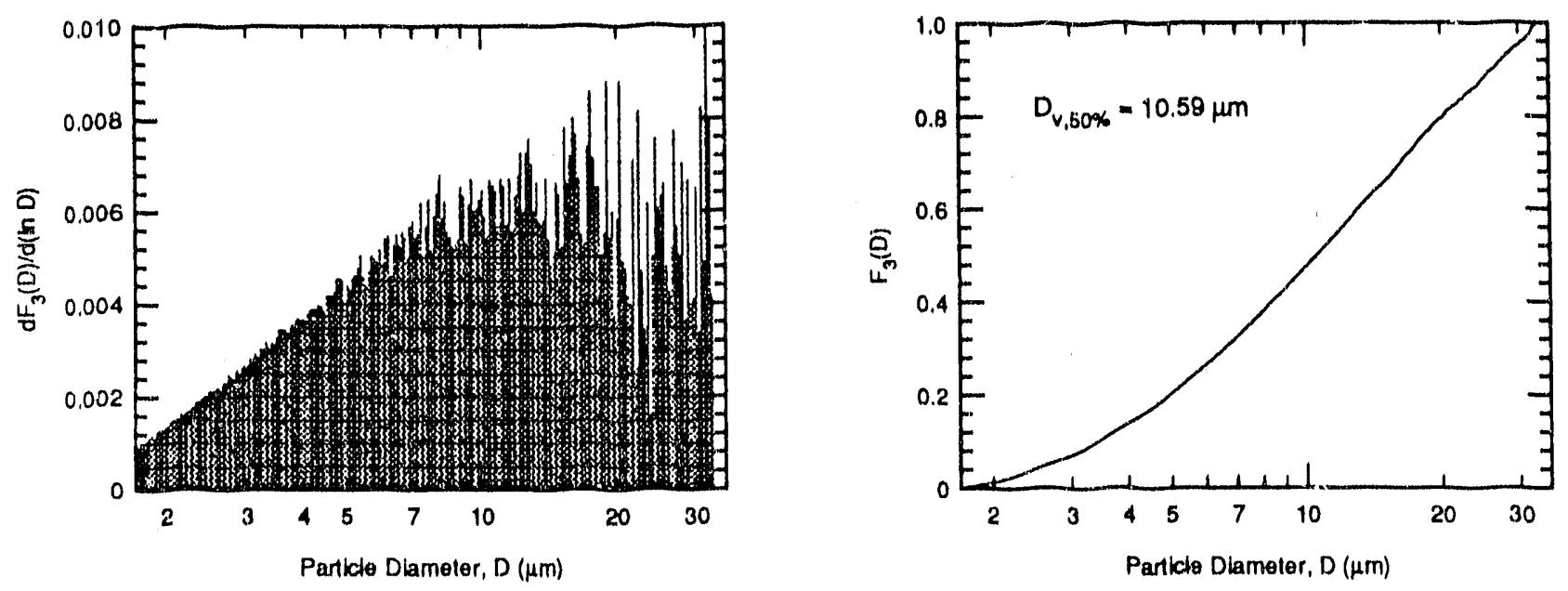

Volume Distribution

Figure 8: Differential and Cumulative Size Distributions for Eagle Butte Ash, Density > $2.2 \mathrm{~g} / \mathrm{cc}$. Multisizer Orifice Dlameter $=50 \mu \mathrm{m}$. Particle Count $=238,471$ 

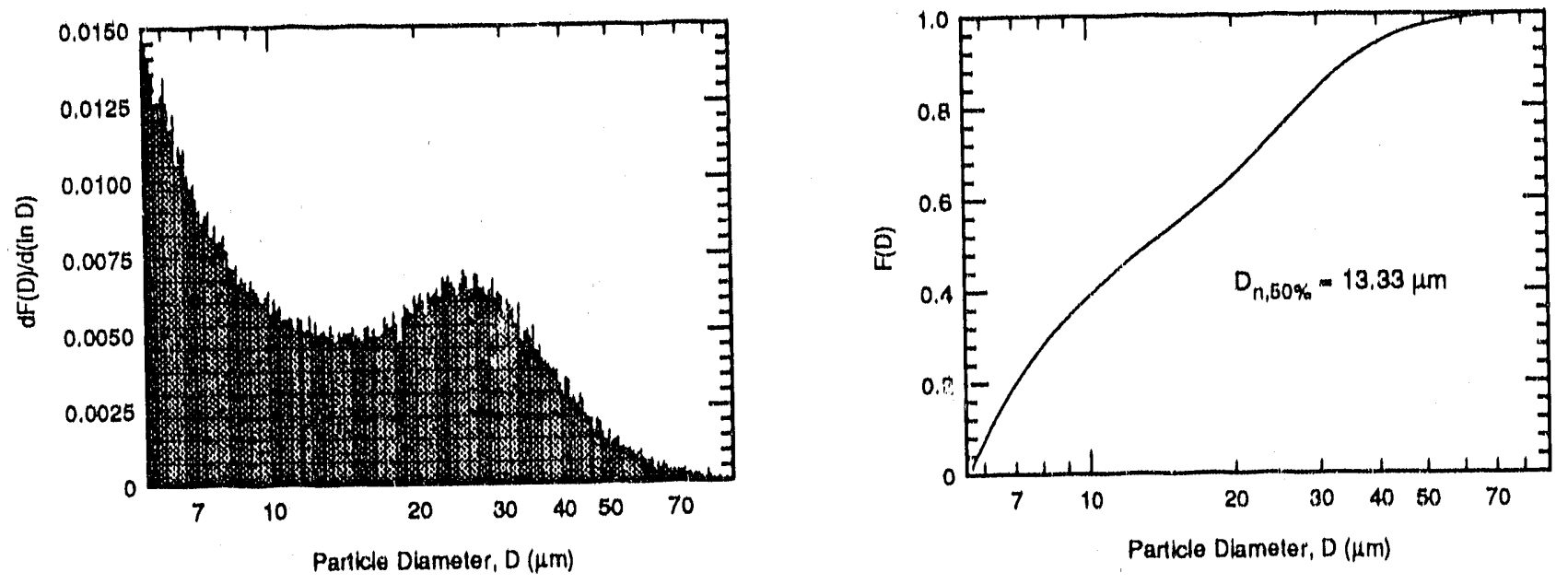

Number/Count Dlstribution
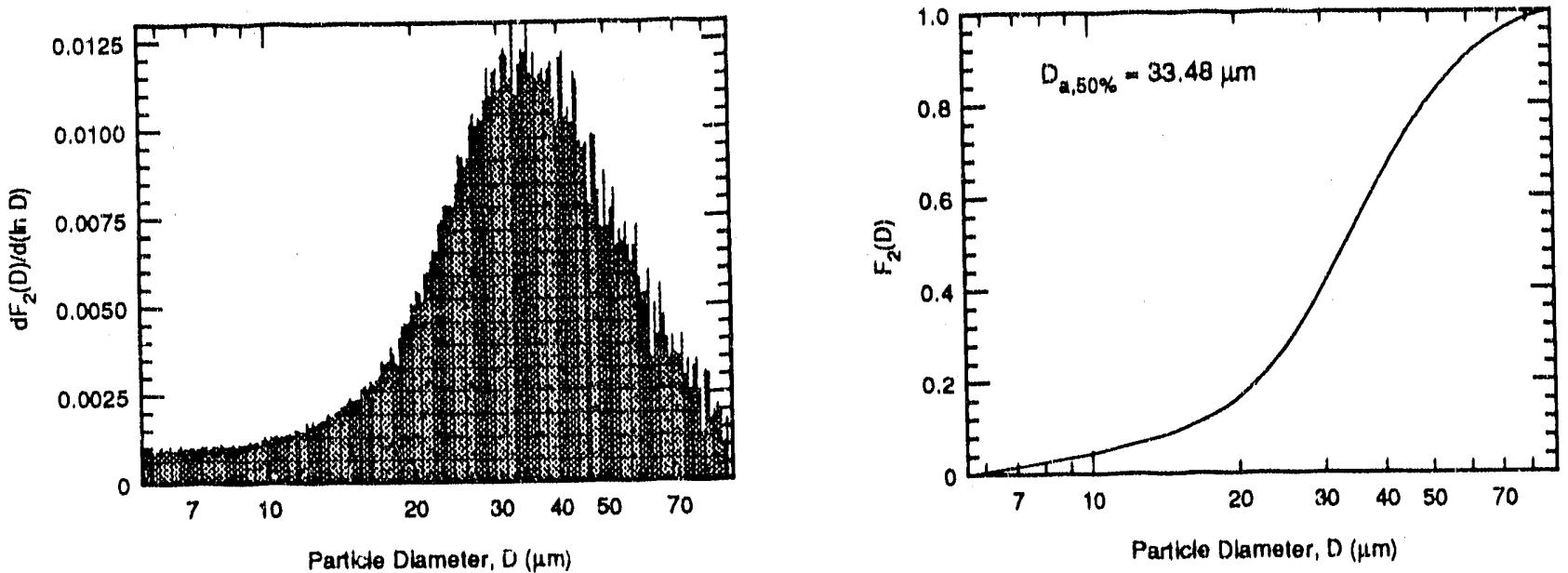

Area Distribution
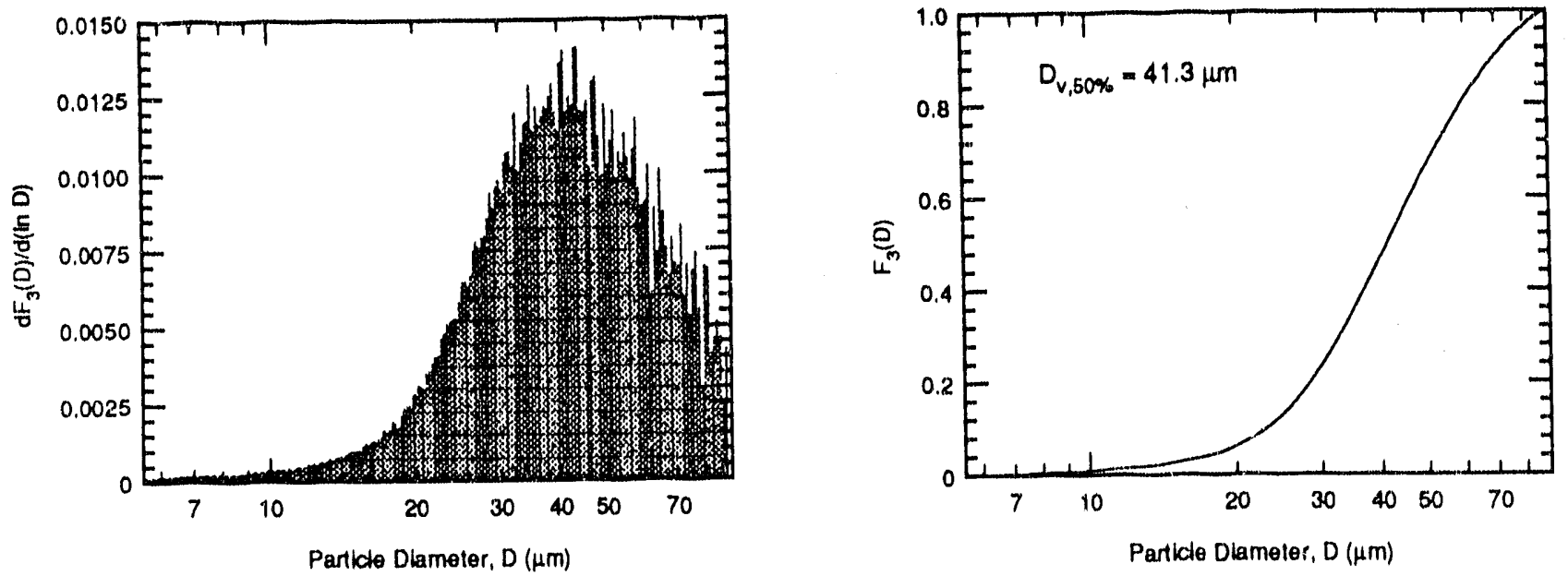

Volume Distribution

Figure 9: Differential and Cumulative Size Distributions for Beulah Baghouse Ash, Density < $2.2 \mathrm{~g} / \mathrm{cc}$. Multisizer Orifice Diameter $=140 \mu \mathrm{m}$. Total Particle Count $=105,145$. 

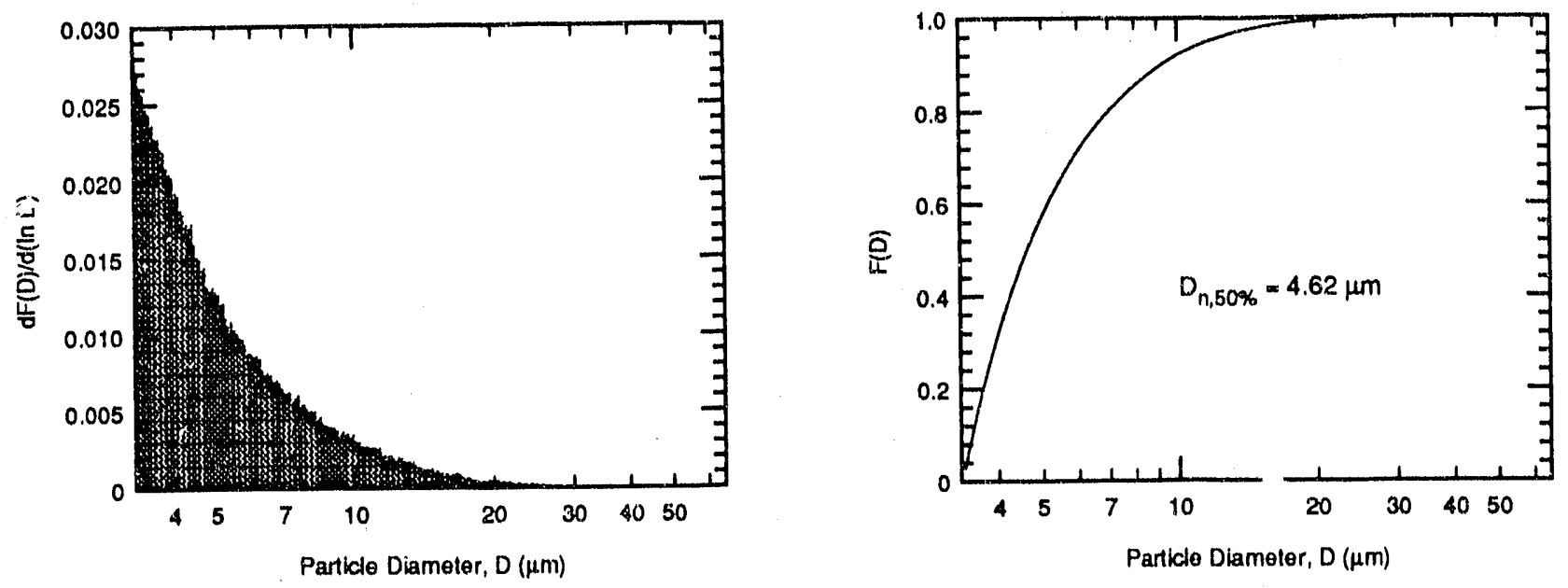

Number/Count Distribution
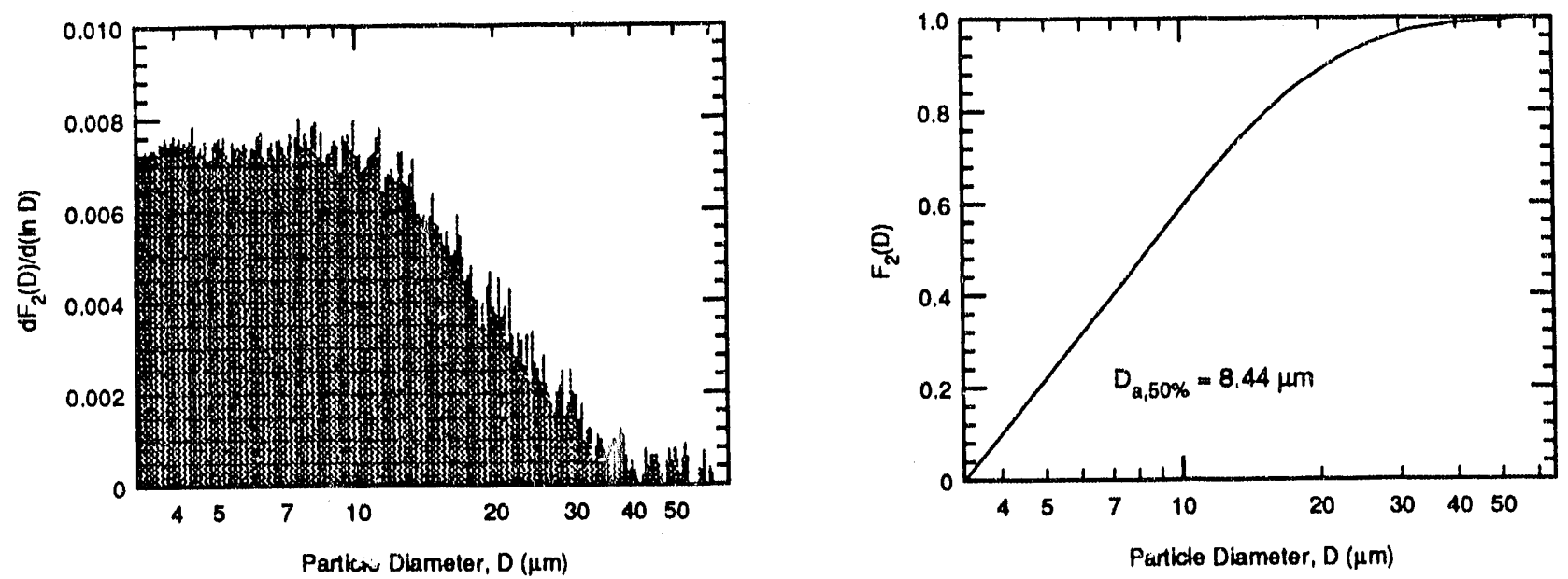

Area Distribution
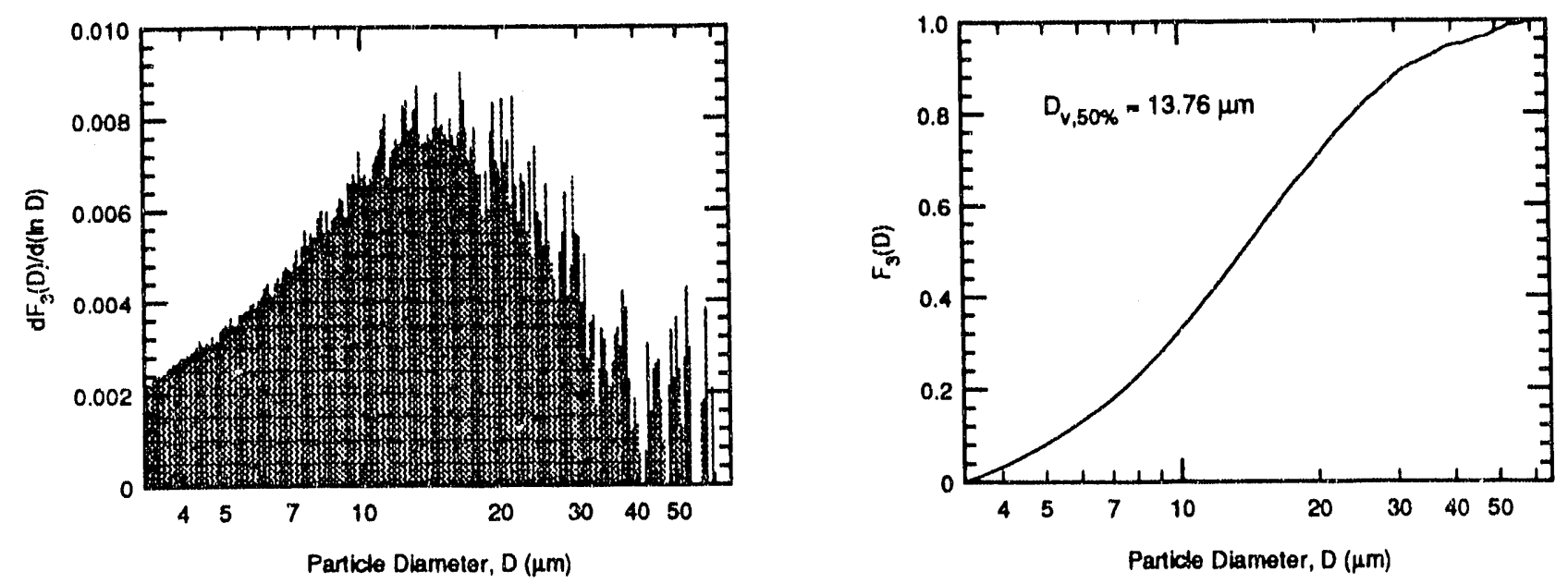

Volume Distribution

Figure 10: Differential and Cumulative Size Distributions for Beulah Baghouse Ash, Density >2.2 g/cc. Multisizer Orifice Diameter $=100 \mu \mathrm{m}$. Total Particle Count $=220,000$ 


\subsection{Task 2: The Optical Properties of High Temperature Slag}

The immediate goal of this task is to measure the high temperature optical properties of coal slag. The complex refractive index, $m=n+i k$, is being measured for synthetic slags at high temperatures, near $1900 \mathrm{~K}$. The effect of composition on the optical properties is also being investigated.

In previous QPR's, measurements of the near normal reflectance of nine slag compositions were reported (SA00, SA01, SA05, SA10, SA20, SA4S, SA2S, SA1S, and NAEB). From those measurements the complex refractive index, $m$, was computed using the Kramers-Kronig relations, with $n$ being determined for the wavelength range $1<\lambda<13 \mu m$ and $k$ being determined for a narrower range $(8<\lambda<13 \mu m)$. These nine slags span a range of compositions similar to those found for most (by volume) ash particles under Task 1.

As reported in a previous (7-1991) QPR, transmittance measurements were completed for three slags (SA01, SA4S, and SA05). The transmittance was measured in the wavelength range $1<\lambda<5 \mu \mathrm{m}$, where the $\mathrm{Fe}_{2} \mathrm{O}_{3}$ in the slag causes absorption of the infrared radiation. These measurements conclude Task 2 , and provide sufficient information for determining the radiative properties of fly ash dispersions.

\subsubsection{Scheduled Activities}

Task 2 is now complete. During the next quarter the final report will be prepared. 


\subsection{Task 3: Sample Calculations of the Radiant Properties of Fly Ash Dis- persions}

The goals of this task are to provide sample calculations which allow estimates of the influence of fly ash on the total radiative heat transfer in coal combustors and provide computational capabilities required to evaluate our overall approach using results from Task 4.

\subsubsection{Overview}

In this research program, we have adopted the approach that by measuring fundamental properties (i.e, the complex refractive index, $m$ ) of the fly ash which participates in the radiation transfer, one can use well established theoretical principles (Mie theory) to compute the radiative properties of dispersions of fly ash as found in coal combustors. With this approach one can understand the underlying principles that affect the radiative properties of an ash dispersion and more confidently predict how variations in the characteristics of the ash dispersion cause variations in its radiative properties.

An important criterion in this approach is that the fly ash particles be spherical, homogeneous, and isotropic. Fortunately, fly ash particles are formed at high temperatures at which most of them are molten, leading primarily to spherical particles. Furthermore, one should expect that molten particles will be reasonably homogeneous and isotropic. On cooling, most fly ash particles form glassy spheres which are homogeneous and isotropic, but some, and perhaps most from some types of coal, may form spheres or spheroids with interior bubbles or voids.

To the extent that some ash particles are not spherical, homogeneous, or even isotropic, one should consider departures from the simpler model as corrections. The importance of these corrections must be weighed in light of the overall influence of ash on radiation heat transfer in coal combustors. We expect that most fly ash is well modeled as isotropic, homogeneous spheres, and that this approach goes much further toward explaining the effects of fly ash on radiative transport than other possible approaches. 


\subsubsection{Progress and Scheduled Activities}

Computer codes were developed to compute the radiative transfer through dispersion of fly ash using data obtained under Task II. During the next quarter the results of these calculation will be prepared for final report. 


\subsection{Task 4: Measurement of Radiant Properties of Fly Ash Dispersion}

In this Task, a bench-scale experiment under controlled conditions in the laboratory is being performed to test the validity of the approach taken in the first three Tasks.

To be specific, the philosophy of our overall approach is the hypothesis that the optical/radiative properties of a particular fly ash dispersion can be predicted by appropriate codes (developed under Task 3) using input data on the size and composition distribution of the ash (determined using appropriate microanalytical techniques in Task 1), together with correlations for the spectral and temperature dependence of the complex refractive index as a function of ash composition (determined under Task 2 from measurements on synthetic slags). This fundamental approach has the advantage that the radiative properties of a particular ash (in a particular coal combustion environment) can be predicted purely by characterizing a sample of the ash using established microanalytical techniques, without having to resort to measurements of the optical/radiative properties of each ash, dispersed in a high temperature environment. Such measurements are extremely difficult (or impossible) to perform in a combustion environment, and it is this fact which led us to adopt the present philosophy.

To test the validity of this approach, Task 4 is intended to compare such predictions with measurements of the optical/radiative properties of a dispersion of a representative ash in a bench-style experiment under well-controlled conditions.

As described in past QPRs, a suitable bench-scale experiment has been designed to satisfy the objective of Task 4. A major design consideration was the conclusion that there is no known method for dispersing and adequately deagglomerating a sample of ash powder into a gas stream to reproduce the conditions of the ash aerosol before it was collected from the exhaust stream of the coal combustor. This conclusion was based on extensive experience of redispersing ash, and other fine, insulating powders into a gas stream for laboratory research in electrostatic precipitation. On the other hand, we found that it is possible to produce stable, well-deagglomerated dispersions of fly ash 
in liquids by using ultrasonic agitation. Moreover, by gentle stirring it is possible to maintain stable liquid dispersions at concentrations much higher than is possible with gaseous dispersions. This fact allows one to perform optical extinction and scattering experiments on a much smaller volume of dispersed ash (contained in an optical test cell) than is possible with a gaseous dispersion.

On the basis of an experiment designed around the use of a liquid dispersion, attention was then focused on the choice of liquids and window material for a test cell to allow extinction and/or scattering experiments over the wavelength range of interest, say $\lambda=1-13 \mu \mathrm{m}$. While there is no liquid that is transparent over the whole range, it was established that by using three separate liquids, namely carbon tetrachloride $\left(\mathrm{CCl}_{4}\right)$, carbon disulfide $\left(\mathrm{CS}_{2}\right)$ and bromoform $\left(\mathrm{CHBr}_{3}\right)$, the full wavelength range could be covered. Moreover, it was also established that there is negligible chemical reaction between fly ash constituents and these liquids. For windows, barium fluoride $\left(\mathrm{BaF}_{2}\right)$ is suitable for the range $\lambda=1-13 \mu \mathrm{m}$.

In the QPR of February 1991, detailed descriptions of the experimental cell were presented. Mie scattering by particles suspended in a liquid medium was discussed. An alternate optical design of a transmissometer, suitable for our experimental conditions where the path length of the infra-red beam is comparable to the beam diameter, was described. Sensitivity calculations for extinction measurements for fly ash dispersed in $\mathrm{CCl}_{4}$ were presented in the QPR of April, 1991. In the previous QPR, tabulated data of the real part of the refractive index $(n(\lambda))$ of bromoform and carbon disulfide were given. In this QPR, results of transmittance measurements on suspensions of Upper Freeport ash in $\mathrm{CCl}_{4}$ are presented.

\subsubsection{Transmittance Measurements on Fly Ash Suspension}

The measured transmittance is the ratio of the signal intensity transmitted through the ash suspension to that through the clean liquid. Initial results in the near-infrared with Upper Freeport ash suspended in $\mathrm{CCl}_{4}$ were presented in the previous QPR. Last quarter, transmittance measurements over the spectral range of 1-6 $\mu \mathrm{m}$ were completed. 
The results are discussed in the rest of this section. Measurements on ash suspended in $\mathrm{CHBr}_{3}$ and $\mathrm{CS}_{2}$ are currently in progress.

Higher loadings $\left(V_{a}=2-3 \times 10^{-4}\right)$ than that reported in last quarter were used to achieve extinctions as high as $60 \%$. As a result, small changes in extinction were clearly noticeable. Figure 11 shows the data for the entire range for $\mathrm{CCl}_{4}(1-6 \mu \mathrm{m})$. Comparison with calculations for ash of a single (slag) composition shows that the transmittance increases with wavelength with a smaller slope in the region 1-3 $\mu \mathrm{m}$ than that predicted from Mie calculations. Beyond $3 \mu \mathrm{m}$, the spectral transmittance becomes significantly flatter, while the calculations predict the transmittance to continue to increase until $\approx 5.6 \mu \mathrm{m}$. Here, the relative refractive index, $n_{\text {rel }}$, passes through unity; at which point the scattering falls to zero, and the small extinction is due entirely to absorption.

To further investigate the discrepancy between the data and the predictions, transmittance measurements were also made on the ash fractions that were density separated with liquid of density $2.2 \mathrm{~g} / \mathrm{cc}$. As discussed under Task 1, the fractions lighter than $2.2 \mathrm{~g} / \mathrm{cc}$ consists almost entirely of cenospheres. The densities of the two fractions, measured with the specific gravity bottle, are $\rho_{<2.2}=2.01 \mathrm{~g} / \mathrm{cc}$ and $\rho_{>2.2}=2.36 \mathrm{~g} / \mathrm{cc}$. Since $23.9 \%$ by mass of the Upper Freeport has $\rho<2.2 \mathrm{~g} / \mathrm{cc}$, the density of the whole ash can be obtained by the following simple calculation

$$
\rho=(0.239)(2.01)+(1-0.239)(2.36)=2.28
$$

This value compares well with the previously determined density of $2.29 \mathrm{~g} / \mathrm{cc}$ (QPR of May, 1991), and serves as a check for the densities measured.

The transmittance data for the cenospheric and non-cenospheric fractions are shown in Figure 12. It is immediately apparent that the transmittance increase rate is significantly smaller for the cenospheric fraction, especially in the wavelength range 1-3 $\mu \mathrm{m}$. The slope of the non-cenospheric fraction is much closer to the calculations than the data for the whole ash, which indicates that the cenospheric fraction in the ash is primarily responsible for the flattening of the transmittance curve in the range $1-3 \mu \mathrm{m}$. In this manner, the cenospheric content of the ash has become more important in Task 4 than 
was anticipated.

While Mie calculations for non-concentric cenospheres with multiple bubbles are formidable to the point of being impractical, approximate engineering models may be formulated to explain the trend seen in the transmittance measurements. Because $\left(n_{\text {rel }}-\right.$ 1 ) is very small, a first approximation is to assume that $n_{r e l}=1$, so that the incident electromagnetic wave 'sees' only the bubble and not the glassy ash. Additionally, it is assumed that the all cenospheres are concentric hollow spheres characterized by a single value of $R_{c}$, the ratio of inner diameter to the outer diameter of the cenosphere. As described in the QPR of April, 1990, this ratio is obtained as

$$
R_{\mathrm{c}}=\sqrt[3]{1-\frac{\rho_{<2.2}}{\rho_{>2.2}}}
$$

By this model, the cenospheres suspended in the liquid is represented by air bubbles with diameters reduced by a factor of $1 / R_{c}$. If we further assume that all the bubbles are large $\left(D \gg \lambda_{\text {rel }}\right)$, then we have $Q_{e}=2[3]$. The last assumption was relaxed by assigning to the bubbles the size distribution of the cenospheric fraction which were described in the section on Task 1. Mie scattering calculations for the air bubbles in the liquid was performed using $n(\lambda)$ determined in the last two quarters.

The results of the calculations are shown in Figure 13 along with the transmittance data. The volume median diameter of the bubbles is given by

$$
D_{v, b}=R_{c} D_{v}=0.53 \times 23.1=12.23 \mu \mathrm{m}
$$

Since $R_{c}$ is the same for all ash particles, the geometric standard deviation of the air bubbles is $\sigma_{g, b}=\sigma_{g}$. Noting the expanded ordinate scale and the unavoidable settling of a small fraction of the ash particles, the first approximation model does a fairly good job of predicting the transmittance. The difference between the Mie calculations and the large sphere model is small, and is maximum where the size parameter for most particles falls in the Mie scattering regime, i.e., $x \sim 1$. A better model is based on Mie calculations for concentric spheres with a constant or variable $R_{c}$. Accounting for the glassy ash should show an increasing trend in transmittance with wavelength. Results will be presented in the next QPR. 
While the presence of cenospheres may explain the difference between transmittance data and predictions for the wavelength range 1-3 $\mu \mathrm{m}$, the key to the explanation of the flat spectral nature of the transmittance in the range 3-6 $\mu \mathrm{m}$ lies in the compositional heterogeneity of the ash. The above calculations assume that particles of all diameters have the same composition. As discussed before, the transmittance of the ash dispersion increases to almost $100 \%$ because the $n_{\text {rel }}$ of all particles reaches a value of unity at the same wavelength. However, as we learnt from the CCSEM data, chemical composition varies significantly from particle to particle. Hence, $n_{\text {rel }}$ reaches unity at different wavelengths for different particles, which results in a flattening of the spectral transmittance. Calculations are currently underway to incorporate this variation of $n_{r e l}$ in our calculations. Results will be reported in the next QPR.

Scheduled Activities: The remaining measurements on the Upper Freeport ash will be completed in the next quarter. The San Miguel and Eagle Butte ashes have been selected for further transmittance measurements. The former ash consists almost entirely of cenospheres while the latter contains less than $5 \%$ cenospheres by mass. Hence, measurements on density separated ashes is not expected to be necessary for these two cases. Work on Task 4 is expected to be completed by the end of next quarter.

\section{References}

[1] Appen, A. A. The Chemistry of Glass, (in Russian), Izd. Chimia, Leningrad, 1970. Translated Data Available in: Volf M. B. Mathematical Approach to Glass, Elsevier, pp. 223 (1988).

[2] Goodwin, D. G. Infrared Optical Constants of Coal Slags, Report T-255, High Temperature Gas Dynamics Laboratory, Stanford University (1986).

[3] van de Hulst, H. C. Light Scattering by Small Particles, Dover Publications, pp. 133 (1957). 


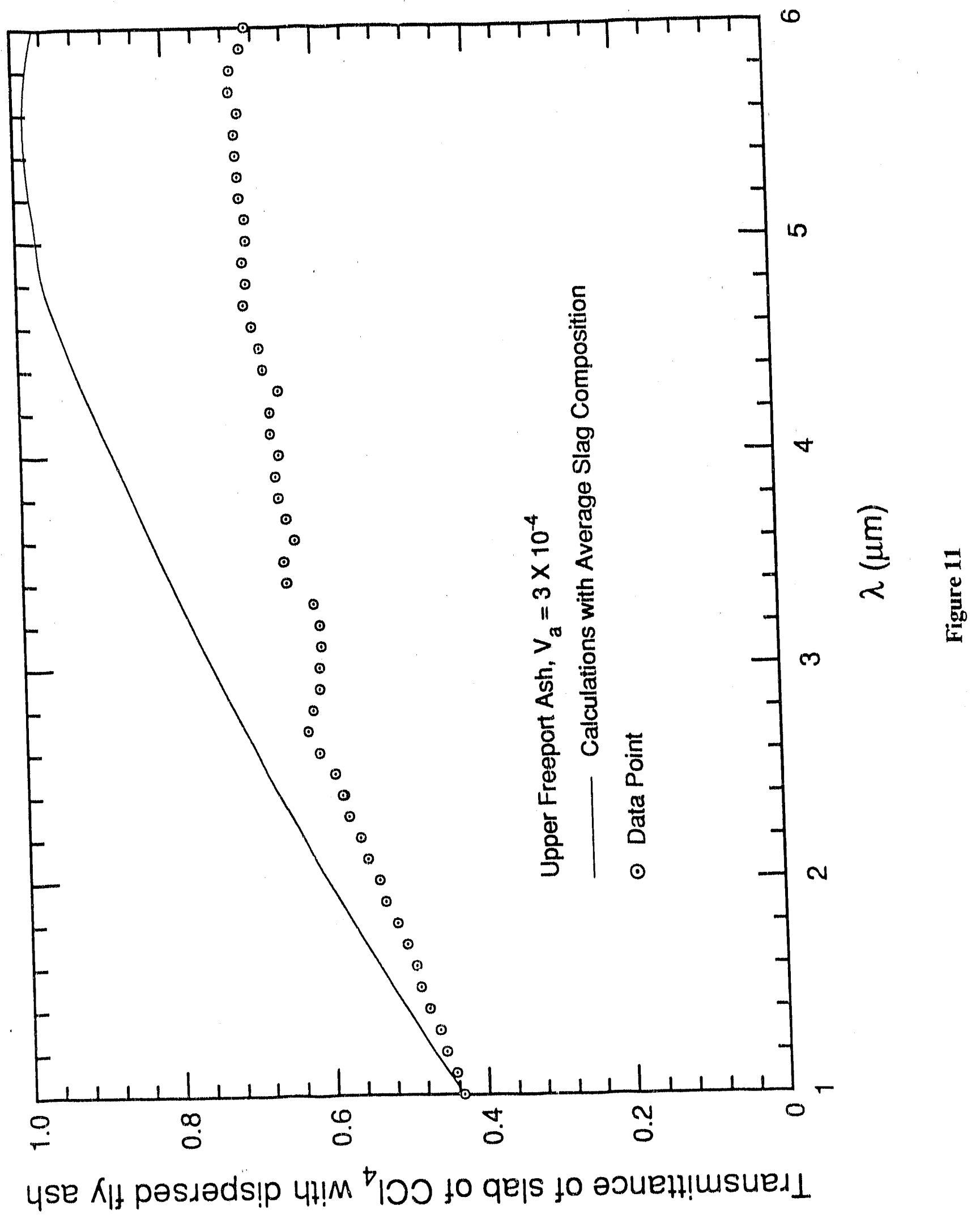




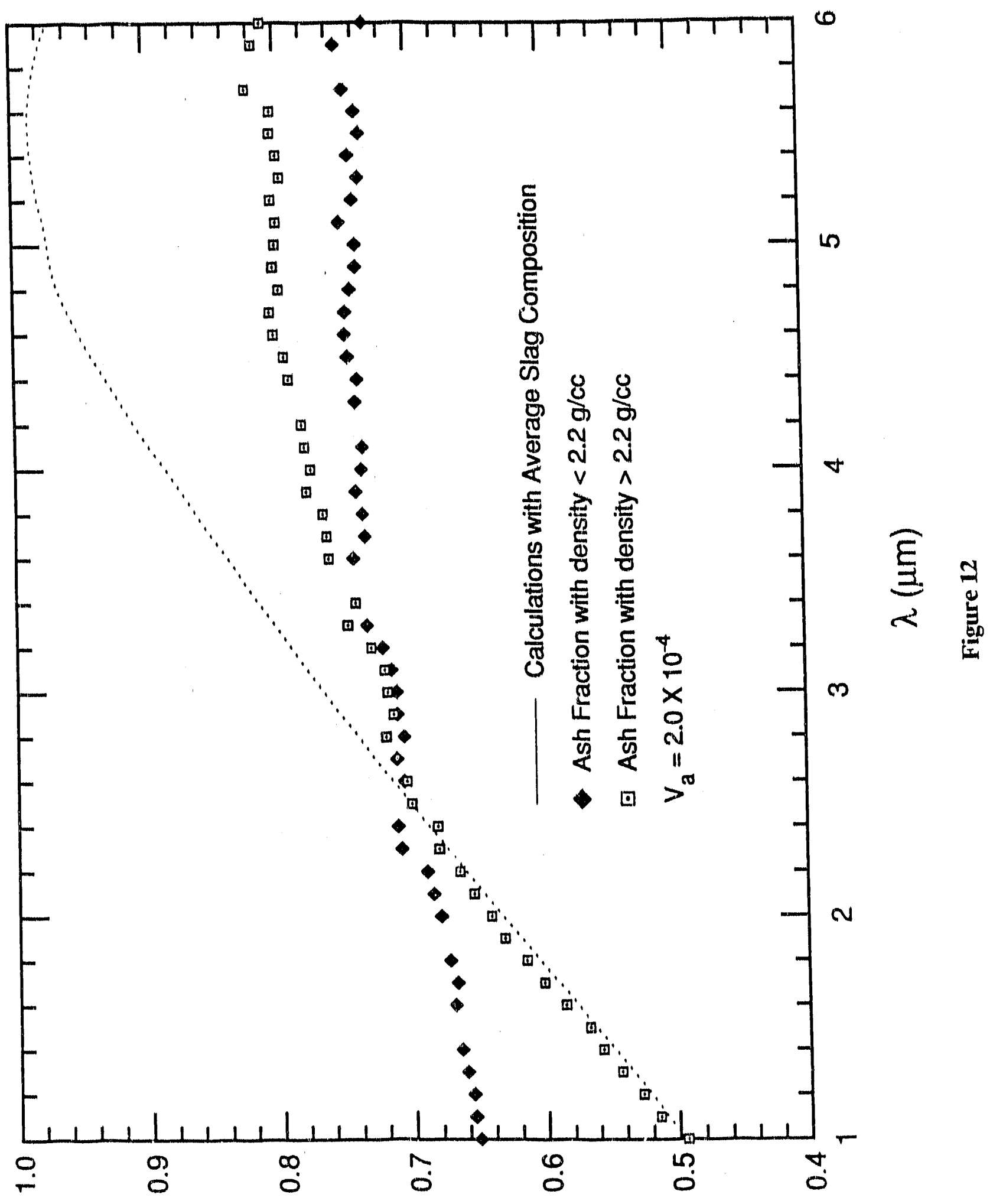

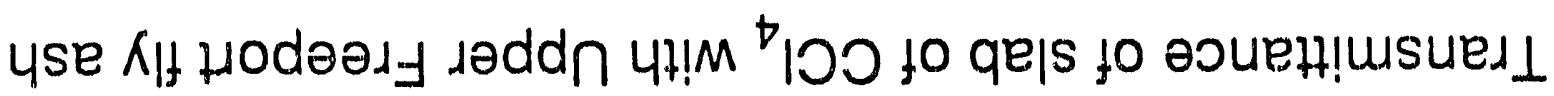




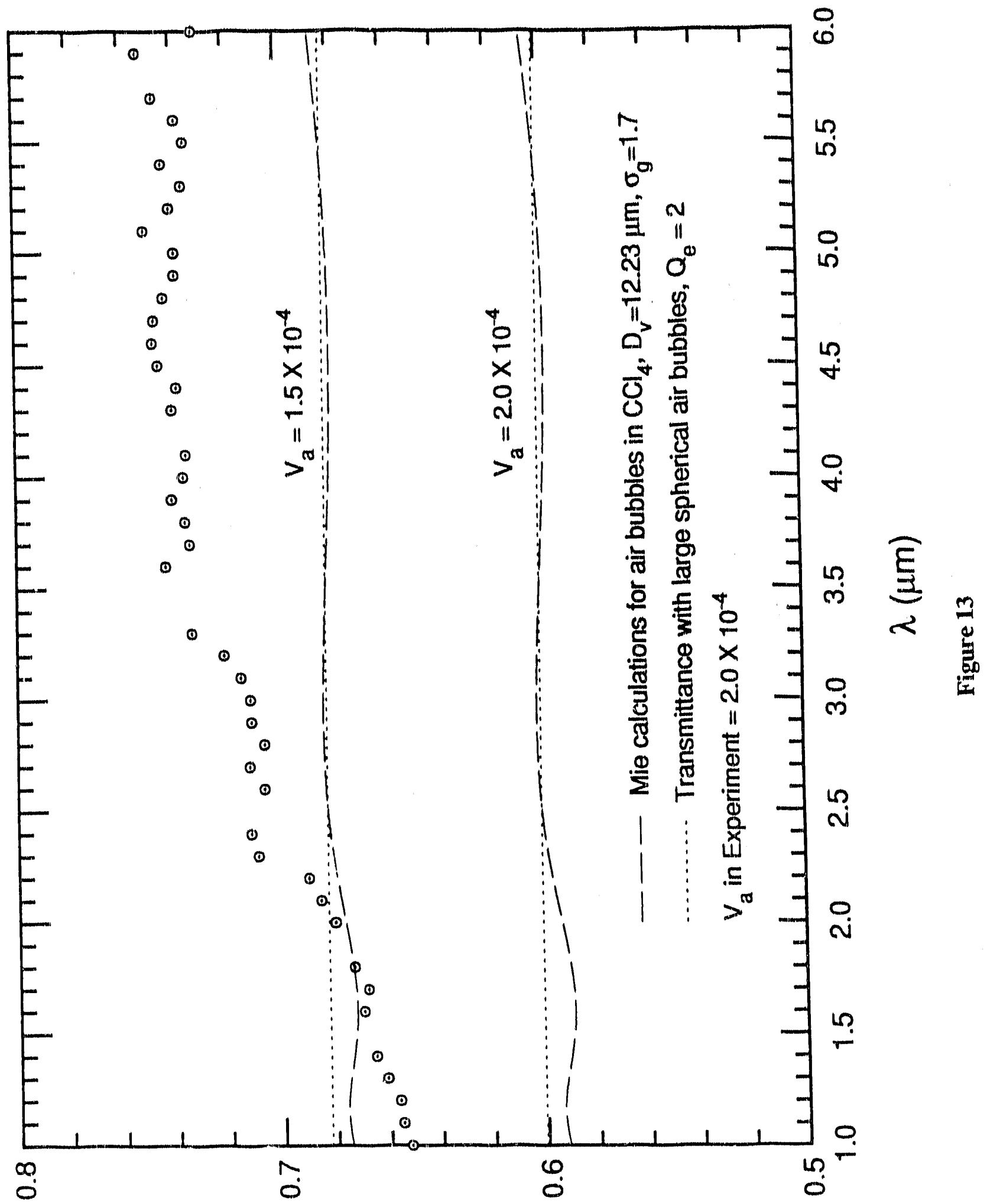

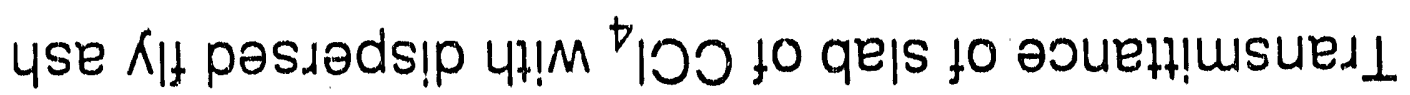



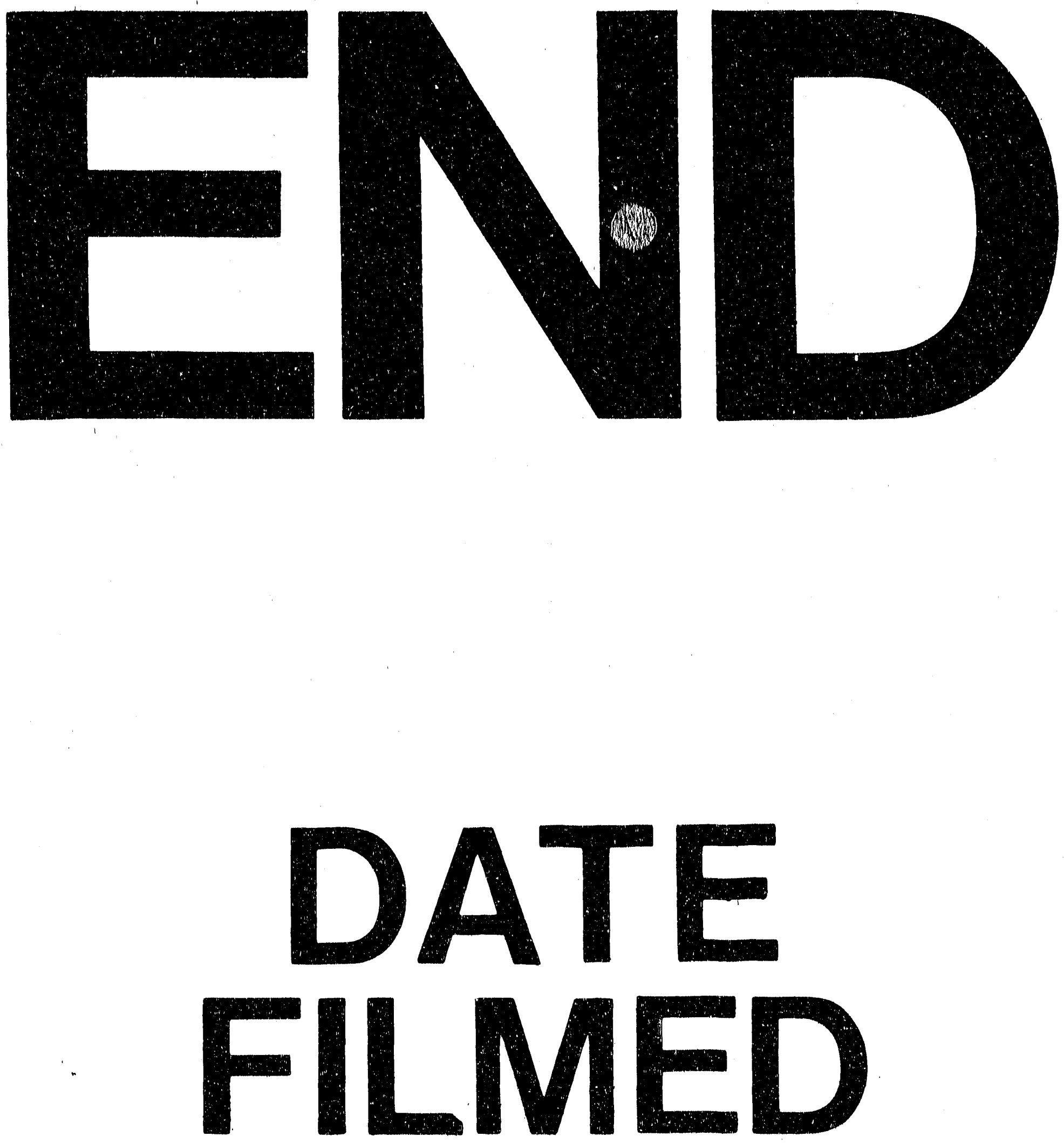

I

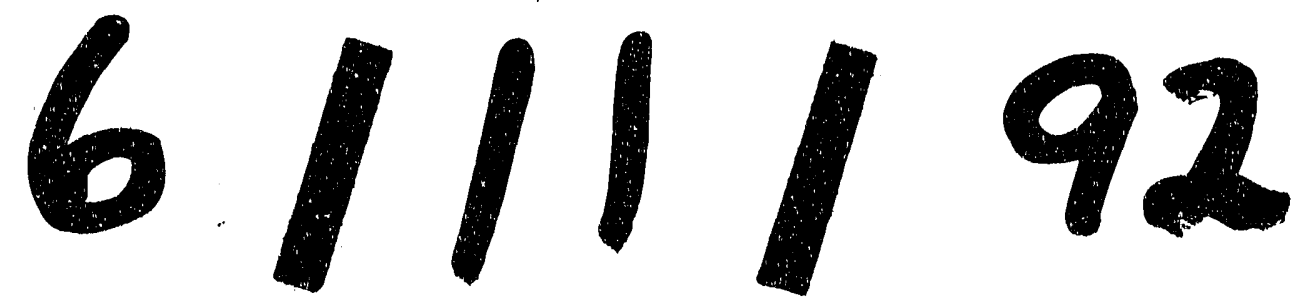


